\title{
1 Transport mechanism of the neuronal excitatory amino acid transporter
}

2

3

4 Biao Qiu ${ }^{1}$, Doreen Matthies ${ }^{2}$, Eva Fortea $^{1}$, Zhiheng Yu², Olga Boudker ${ }^{1,3 *}$

5

6

$7 \quad{ }^{1}$ Department of Physiology \& Biophysics, Weill Cornell Medicine, 1300 York Ave, New York, $8 \quad$ NY 10021, USA

$9{ }^{2}$ Howard Hughes Medical Institute, Janelia Research Campus, 19700 Helix Drive, Ashburn, VA 10 20147, USA

$11{ }^{3}$ Howard Hughes Medical Institute, Weill Cornell Medicine, 1300 York Ave, New York, NY 12 10021, USA

13

$14 *$ Corresponding author 


\section{Abstract}

Human excitatory amino acid transporter 3 (hEAAT3) mediates glutamate uptake in neurons, intestine, and kidney. Here, we report Cryo-EM structures of hEAAT3 in several functional states where the transporter is empty, bound to coupled sodium ions only, or fully loaded with three sodium ions, a proton, and the substrate aspartate. The structures suggest that hEAAT3 operates

21 by an elevator mechanism involving three functionally independent subunits. When the substrate-

22 binding site is near the cytoplasm, it has a remarkably low affinity for the substrate, perhaps

23 facilitating its release and allowing for the rapid transport turnover. The mechanism of the coupled

24 uptake of the sodium ions and the substrate is conserved across evolutionarily distant families and is augmented by coupling to protons in EAATs. The structures further suggest a mechanism by which conserved glutamate mediates proton symport.

\section{Introduction}

29 Human excitatory amino acid transporters (hEAATs) pump glutamate into cells against steep concentration gradients by utilizing the pre-established transmembrane gradients of sodium and potassium ions and protons as an energy source ${ }^{1,2}$. hEAAT3 is one of five human EAAT subtypes

32 broadly expressed in neurons throughout the brain. It is the major glutamate and aspartate transporter outside of the central nervous system (CNS), including in kidneys and intestines ${ }^{3-5}$. Mutations in hEAAT3 cause dicarboxylic aminoaciduria, a metabolic disorder that leads to the excessive loss of aspartate and glutamate in the urine ${ }^{6}$. Notably, the disorder can be associated with mental retardation and obsessive-compulsive syndrome ${ }^{7}$. The causative role of EAAT3 loss was confirmed using knockout mice, which also developed dicarboxylic aminoaciduria ${ }^{8}$.

In the mammalian brains, EAAT3 is expressed in the somata and dendrites of both excitatory and inhibitory neurons but excluded from presynaptic membranes. EAAT3 total levels in the brain are approximately 100 times lower than EAAT2 ${ }^{9}$, which mediates most of the glutamate uptake

42 following synaptic transmission ${ }^{10}$. Nevertheless, altered EAAT3 expression and plasma membrane

43 trafficking are associated with epilepsy, schizophrenia, hypoxia, and decreased activity, which

44 leads to a susceptibility of neurons to oxidative damage, particularly in epilepsy models ${ }^{11-14}$. The 45 relevance of EAAT3 to oxidative stress resistance might be due to its ability, unique among 46 EAATs, to transport cysteine, which is a rate-limiting intermediate in the biosynthesis of the 
47 antioxidant glutathione ${ }^{15-17}$. Thus, activators of hEAAT3 might be useful to boost cysteine uptake 48 into neurons under pathologic conditions. Due to the high concentration of glutamate in neurons, 49 hEAAT3 might also run in reverse during ischemia, spilling excitotoxic levels of glutamate into

50 the extracellular space. Therefore, specific inhibitors of hEAAT3 might be clinically helpful in 51 reducing glutamate-mediated damage following transient ischemic conditions and depolarization 52 of membranes.

54 A detailed understanding of the hEAAT3 structure and mechanism would be required to enable 55 pharmacologic manipulations. Toward this end, we have determined structures of hEAAT3 using 56 Cryo-EM. We show that the transporter is a homotrimer. Each protomer consists of two domains, 57 a central trimerization scaffold domain and a peripheral transport domain containing the substratebinding site. The individual hEAAT3 protomers undergo elevator-like transitions between the outward- and inward-facing conformations, in which their transport domains localize closer to the extracellular space and the cytoplasm, respectively. They appear to be independent of each other so that we observe trimers with every possible arrangement of the outward- and inward-facing protomers. Remarkably, when we imaged hEAAT3 in the presence of L-aspartate (L-asp) and sodium $\left(\mathrm{Na}^{+}\right)$ions, we observe that only the outward-facing protomers were bound to the amino acid, while the inward-facing protomers remained substrate-free. We conclude that the substratefree transporter has a strong preference for the inward-facing conformation while the outwardfacing transporter has a significantly higher affinity for the amino acid. By comparing the structures of the transport domain when free of ligands, bound to $\mathrm{Na}^{+}$ions only and bound to $\mathrm{Na}^{+}$ ions, protons, and L-asp, we provide mechanistic insights into symport of the amino acid, $\mathrm{Na}^{+}$and protons in hEAAT3.

\section{Results}

72 The elevator mechanism of hEAAT3. We heterologously expressed and purified full-length 73 hEAAT3 with two potential glycosylation sites mutated (N178T and N195T), hEAAT3g (ED

74 Figure 1a - c). The wild type and mutant showed L-asp uptake in oocytes with a $K_{m}$ of $39 \pm 7$ and $7563 \pm 11 \mu \mathrm{M}$ (Figure 1a), respectively. The purified, liposome-reconstituted hEAAT3g showed 76 electrogenic L-asp and L-glutamate (L-glu) uptake in solid-supported membrane 77 electrophysiology assays (ED Figure 1d). The uptake was dependent on a $\mathrm{Na}^{+}$ion gradient and 
78 inhibited by (3S)-3-[[3-[[4-(Trifluoromethyl)benzoyl]amino]phenyl]methoxy]-L-aspartic acid

79 (TFB-TBOA) (ED Figure 1e). The $K_{m}$ values for L-asp and L-glu $(242 \pm 24$ and $883 \pm 120 \mu \mathrm{M}$,

80 respectively) (Figure 1b) were higher than in oocytes and mammalian cells ${ }^{18-20}$. The discrepancies

81 might reflect distinct lipid environments or be due to mixed orientations of the hEAAT3 ${ }_{\mathrm{g}}$ proteins

82 reconstituted into the supported bilayers. Indeed, hEAAT3 showed a significantly higher $K_{m}$ of

83 reverse transport ${ }^{21,22}$.

We first acquired Cryo-EM images of hEAAT3 ${ }_{\mathrm{g}}$ in the presence of $200 \mathrm{mM} \mathrm{NaCl}$ and $1 \mathrm{mM} \mathrm{L-}$ asp. 2D and 3D classifications were carried out to select a relatively homogenous subset of particles. 3D refinement and reconstruction on the subset with $\mathrm{C} 3$ symmetry yielded an electron density map with an overall resolution at $2.85 \AA$ (Table 1, ED Figure 2a-c and ED Figure 3b). The trimeric transporter showed an overall architecture (ED Figure 4a) similar to those of the homologous archaeal transporters Glt $\mathrm{Ph}_{\mathrm{P}}$ and $\mathrm{Glt}_{\mathrm{Tk}}{ }^{23-26}$, a thermostabilized variant of human EAAT1 $\left(\right.$ htsEAAT1) ${ }^{27}$, and human neutral amino acid transporter ASCT2 ${ }^{28,29}$. The central trimerization scaffold supported three peripheral transport domains in inward-facing states. Consequent symmetry expansion revealed that $\sim 19 \%$ of the protomers were in an outward-facing conformation (ED Figure 2, 3a). By sorting particle images of 554,920 EAAT3 trimers based on the conformations of their protomers, we estimated the fractions containing three, two, one, and zero inward-facing protomers, which were in good agreement with the binomial distribution (Figure 1c). Thus, transport domains sample the outward- and inward-facing orientations independently of each other, consistent with the body of literature ${ }^{30-32}$.

We refined the inward-facing symmetric trimer and the asymmetric trimer with two protomers in 101 the inward-facing state and one protomer in the outward-facing to 3.03 and $3.42 \AA$ resolution, 102 respectively (Table 1, ED Figure 2d and ED Figure 3d). The map obtained using all particles 103 was virtually indistinguishable from the map based on symmetric inward-facing trimers only. 104 However, it reached a higher resolution, and we used it for further protein model building. We also 105 refined 7,737 symmetric outward-facing trimers and the single outward-facing protomer to $3.69 \AA$ 106 and $3.03 \AA$ resolution, respectively (Table 1, ED Figures 2e and ED Figure 3a, c). 
In the outward- and inward-facing protomers, the scaffold domain remained mostly unchanged. In contrast, the transport domain moved by $\sim 20 \AA$ from an extracellular to an intracellular position enabled by the inter-domain hinges (Figure 1d). Most remarkably, the outward-facing protomers

111 were bound to L-asp (OFS-Asp), while the inward-facing protomers were not (Figure 1d). In the 112 inward-facing protomers (IFS- $\mathrm{Na}^{+}$), $\mathrm{Na}^{+}$ions are likely bound in at least one of the sites (discussed

113 below), and we observe a characteristic opening of the helical hairpin 2 (HP2) proposed to gate 114 the substrate-binding site ${ }^{33}$. When we imaged hEAAT3 $\mathrm{g}$ in $20 \mathrm{mM}$ L-asp, the population of the 115 OFS-Asp protomers increased to $\sim 62 \%$, but the inward-facing protomers remained mostly 116 unbound (ED Figure 5a, ED Figure 7, and Table 2). Finally, in 10 mM L-glu, we observed only 117 substrate-free IFS-Na ${ }^{+}$protomers (ED Figure 5b, ED Figure 7, and Table 2).

119 These results suggest that in the absence of transmembrane electrochemical gradients and substrate, 120 hEAAT3 $_{\mathrm{g}}$ strongly favors the inward-facing conformation, similar to the archaeal homologue $121 \mathrm{Glt}_{\mathrm{Tk}}{ }^{34}$. In this state, at least under our imaging conditions, the transporter has a remarkably low 122 substrate affinity, perhaps in tens of mM. Comparing inward-facing hEAAT3 ${ }_{g}$ to ASCT2, we 123 observed that in substrate-free proteins, the transport domain leans away from the scaffold, while 124 HP2 remains in contact with the scaffold (Figure 1e). Thus, the binding site is open to the 125 cytoplasm. Upon substrate binding to ASCT2, the tip of HP2 closes over the binding pocket. We 126 expect that substrate binding and occlusion in hEAAT3 $\mathrm{g}$ occur by a similar mechanism. However, 127 the HP2 tip is more extensively engaged with the scaffold in substrate-free hEAAT3 $3_{\mathrm{g}}$ than in $128 \operatorname{ASCT}^{28,33}$ (Figure 1e), perhaps explaining, at least in part, its low affinity for the substrate.

130 Distinct mode of substrate-binding to $\boldsymbol{h E A A T 3 . ~ T h e ~ s u b s t r a t e - b i n d i n g ~ s i t e ~ g e o m e t r y ~ i n ~} \mathrm{hEAAT} \mathrm{g}_{\mathrm{g}}$ 131 OFS-Asp resembles that of the archaeal homologs and htsEAAT1, but L-asp side-chain takes a 132 different rotamer and is coordinated differently in hEAAT3 (Figure 2a, b, ED Figure 5c). The 133 main-chain carboxylate of L-asp is coordinated principally by N451 401 (here and elsewhere, the 134 corresponding residue number in Gltph is shown as the subscript for reference) in the 135 transmembrane segment (TM) 8 and S333 278 in HP1, similar to other homologs ${ }^{24,27}$. In contrast, 136 the side-chain carboxylate and the amino group of L-asp are coordinated differently. Carboxyl 137 oxygens (OD1 and 2) form hydrogen bonds with R447397 in TM8, the main-chain amides of HP2 138 (OD1), and $\mathrm{T}_{370_{314}}$ in TM7 (OD2) in the archaeal transporters and htsEAAT1. In hEAAT3, the 
carboxyl group and R447 397 face away from each other somewhat. While still hydrogen-bonded by the guanidinium group, the carboxylate appears to be more engaged by $\mathrm{T}_{370_{314}}$ (Figure 2a).

141 L-asp amino group in other homologs is coordinated primarily by the highly conserved D444394 in

142 TM8, but in hEAAT3 $\mathrm{g}$, it is coordinated only by the carbonyl oxygens in HP1 and HP2. D444 394 143 does not interact with L-asp and, instead, interacts with R447397. The structural basis of why the 144 substrate binds differently to hEAAT $3_{\mathrm{g}}$ remains unclear because the residues forming the binding 145 site are highly conserved and essential for transport ${ }^{20,35,36}$. Reduced reliance on R447 397 and 146 coordination of the arginine by D444394 might explain why hEAAT3 accepts cysteine as a substrate 147 while the other homologs do not.

$\mathrm{Na}^{+}$and substrate coupling. We observed density corresponding to $\mathrm{Na}^{+}$ions at all three conserved $150 \mathrm{Na}^{+}$-binding sites $\mathrm{Na1}, 2$, and 3 in the OFS-Asp protomer (ED Figure 5d). In the IFS-Na 151 protomers, L-asp and Na2 sites are distorted and empty because of the open conformation of HP2. 152 The coordinating groups of the Nal site, located below the substrate-binding site, are in place, but 153 we did not observe any density for the ion. Moreover, it appears that the site, in particular, the 154 critical D455405 residue, is solvent-accessible (Figure 2c). Although the density for $\mathrm{Na}^{+}$ion in the 155 Na3 site is unresolved, all coordinating residues are in place, and the site is likely occupied (Figure 156 2d). These results suggest that the Na3 site has the highest affinity for the ions, and might be the 157 first to bind, as previously suggested ${ }^{26}$. In contrast, the Na1 site might remain only partially 158 occupied at $200 \mathrm{mM} \mathrm{Na}^{+}$ions used during grid preparation and might be in rapid exchange with 159 the bulk solution.

161 To explore the structural underpinning of the coupled substrate and $\mathrm{Na}^{+}$ion symport, we imaged 162 hEAAT3 $_{\mathrm{g}}$ in the absence of $\mathrm{Na}^{+}$ions and amino acids in buffer containing $100 \mathrm{mM}$ choline 163 chloride (apo state). Following data processing, we only observed inward-facing symmetric 164 trimers (IFS-Apo), which we refined to $3.03 \AA$ resolution (Table 1, ED Figure 3e). The overall 165 architecture of IFS-Apo is similar to IFS-Na ${ }^{+}$, but the transport domain swings further away from 166 the scaffold and shifts outward slightly. The transport domains superimposed with an overall 167 r.m.s.d. of $1.3 \AA$, with the most significant conformational changes occurring in TM7 and HP2 168 (Figure 2e). In further considering the coupling mechanism, we took advantage of the observations that the conformational changes underlying ion and substrate binding are confined to transport 
170 domains and similar in the outward- and inward-facing states of glutamate transporters ${ }^{34,37,38}$. We, 171 therefore, compared the transport domains of IFS-Apo, IFS-Na ${ }^{+}$, and OFS-Asp to visualize 172 structural events during binding.

173

174 A critical structural change upon binding of $\mathrm{Na}^{+}$ions to the apo protein is the repositioning of $175 \mathrm{M} 367_{311}$, part of the highly conserved NMD motif in TM7. When $\mathrm{Na}^{+}$ions bind to Na1 and Na3 176 sites, coordinated, in part, by N366 310, M367 311 sidechain flips from pointing into the lipid bilayer 177 to pointing toward the substrate-binding site, where it also contributes to the $\mathrm{Na} 2$ site (Figure 2e) ${ }^{26}$. 178 The flipped-out M367311 sidechain, as seen in IFS-Apo, would sterically clash with the HP2 179 conformation observed in the OFS-Asp, perhaps ensuring that L-asp does not bind in the absence 180 of the ions (Figure 2e, f). Once $\mathrm{Na}^{+}$ions bind to $\mathrm{Na} 1$ and $\mathrm{Na} 3$ sites, M367311 and HP2 are 181 repositioned in a manner compatible with substrate binding, which causes only minimal further 182 structural changes, limited to the closure of the HP2 tip and the formation of the Na2 site (Figure 183 2f). Similar movements of $\mathrm{M} 367_{311}$ contribute to the coupled binding of $\mathrm{Na}^{+}$ions and L-asp in

$184 \mathrm{Glt}_{\mathrm{Ph}}{ }^{24,37}$, suggesting that the mechanism is conserved in glutamate transporters of vastly different 185 evolutionary origins.

187 Interestingly, in contrast to apo Glt $\mathrm{Ph}_{\mathrm{P}}$ and Glt $\mathrm{Tk}$, in which HP2 can close and occlude the substrate188 binding site, HP2 remains open in hEAAT3 $3_{\mathrm{g}}$. Closure of HP2 is required for the rapid return of the 189 transport domain into the outward-facing state. In human EAATs, coupled to potassium counter190 transport, potassium binding is likely necessary to close HP2. We speculate that the inability of

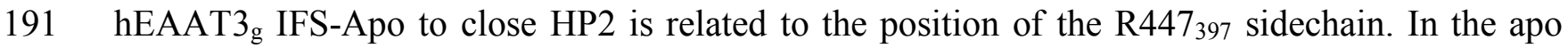
192 archaeal transporters, R447397 moves into the substrate-binding site where it can form direct or 193 through-water hydrogen bonds with the main-chain carbonyl oxygens of the HP2 tip (ED Figure 194 6). In hEAAT3 $3_{g}$ IFS-Apo, R447397 is engaged by a TM7 residue E374318 (glutamine in archaeal 195 homologs) and is not available to close HP2. Unfortunately, we were unable to visualize potassium 196 binding to $\mathrm{hEAAT3}$ gecause the Cryo-EM structure obtained in the presence of $250 \mathrm{mM} \mathrm{KCl}$ 197 looked identical to IFS-Apo (not shown).

199 Gating coupled to proton binding. The working cycle of EAATs involves the symport of a proton 200 along with the amino acid and $\mathrm{Na}^{+}$ions. E374 318 in EAAT3 and the equivalent residues in other 
201 EAATs might be the proton acceptors, and mutating them to glutamines resulted in substrate 202 translocation only in an exchange mode ${ }^{39}$. Consistently, in archaeal homologs and ASCT2, which 203 are not proton-coupled, E374 318 is replaced by a glutamine. Furthermore, mutating R447397 in

204 EAAT3 to cysteine abolished aspartate and glutamate transport, but allowed cysteine transport in 205 an exchange mode, suggesting that R447 is also involved in proton coupling ${ }^{35}$. In OFS-Asp 206 hEAAT3 $_{\mathrm{g}}, \mathrm{E} 374_{318}$ is occluded from the solution by HP2 and makes no interactions with other 207 residues (Figure 3a, d). In IFS-Na ${ }^{+}, \mathrm{HP} 2$ is open, and $\mathrm{E} 374_{318}$ is positioned at the far end of the 208 cavity, where it forms a hydrogen bond with T418362. R447397 interacts with D444394 and, through 209 a cation- $\pi$ interaction, Y373317, as it does in the OFS-Asp (Figure 3b, e). Finally, in IFS-Apo, the 210 sidechains of R447 397 and E374 318 flip towards each other to form a salt bridge (Figure 3c, f). The 211 interaction between R447 397 and Y373 317 breaks and R447 397 descends into the substrate-binding 212 site. We calculated the $\mathrm{pK}_{\mathrm{a}-\mathrm{s}}$ of $\mathrm{E} 374_{318}$ in the apo, $\mathrm{Na}^{+}$-bound, and L-asp-bound states using 213 PROPKA $^{40}$. The obtained values of 7.7, 7.5, and 8.5, respectively, were similar to the 214 experimentally measured $\mathrm{pK}_{\mathrm{a}-\mathrm{s}}$ for proton binding to EAAT3 ${ }^{21}$. Increased $\mathrm{pK}_{\mathrm{a}}$ values and occlusion 215 from solvent suggest that E374 318 is more likely to bind protons as first $\mathrm{Na}^{+}$ions and L-asp bind 216 to the transporter. In this manner, binding of protons, $\mathrm{Na}^{+}$ions, and the substrate are coupled. 217 During the return of the apo transport domain into the outward-facing state, the interactions 218 between $\mathrm{E} 374_{318}$ and $\mathrm{R} 447_{397}$ would allow the occlusion of the deprotonated residue.

\section{Discussion}

220 EAAT3 is the fastest EAAT subtype with turnover times of $\sim 10 \mathrm{msec}$. Like other members of the

221 family, it operates by an elevator mechanism. The movements of the individual domains within 222 the trimer are independent of each other, leading to a stochastic distribution of the outward- and 223 inward-facing protomers. Interestingly, we find that the affinity of the inward-facing state is 224 remarkably low, such that even at $20 \mathrm{mM}$ L-asp, we only observe a very week substrate-like 225 density. In contrast, the substrate affinity of the outward-facing state must be considerably higher, 226 and all outward-facing protomers showed bound L-asp. In that regard, the hEAAT $3_{\mathrm{g}}$ differs from 227 the archaeal Glt ${ }_{\mathrm{Ph}}$, which showed comparable L-asp affinities in the outward- and inward-facing 228 states $^{41}$. Thus, our data suggest that during the transport cycle of EAAT3, the inward-facing 229 substrate-bound closed state is a high-energy transient state and that the release of the substrate is 230 rapid. We speculate that also potassium-bound inward-facing closed conformation might be a 
231 high-energy state, perhaps explaining why we did not observe it in our imaging experiments. This

232 hypothesis is consistent with the reorientation of the potassium-bound transport domain being the

233 rate-limiting step of the transport cycle ${ }^{42}$.

235 Collectively, our data, together with the published work, suggest that a two-prong structural

236 mechanism of $\mathrm{Na}^{+}$and amino acid symport is conserved in evolution from archaea to humans.

237 First, binding of $\mathrm{Na}^{+}$ions to the $\mathrm{Na} 1$ and $\mathrm{Na} 3$ sites is a prerequisite for the binding of substrate

238 and $\mathrm{Na}^{+}$in the $\mathrm{Na} 2$ site and leads to movements of two critical residues: $\mathrm{R} 447397$ out of the binding

239 site to make space for the amino acid, and M367 311 into the binding site, where it coordinates $\mathrm{Na}^{+}$

240 in the $\mathrm{Na} 2$ site. Second, uncoupled transport of $\mathrm{Na}^{+}$ions bound to $\mathrm{Na} 1$ and $\mathrm{Na} 2$ sites is prevented

241 because the closure of the HP2 tip, essential to allow translocation, requires binding of the substrate

242 and $\mathrm{Na}^{+}$in the $\mathrm{Na} 2$ site. In EAATs, the coupling to E374 318 protonation enhances both steps of the

243 mechanism. Breaking of the interaction between E374 318 and R447397, favored by E374 318

244 protonation, is necessary to make the arginine available to coordinate the substrate. Furthermore,

245 the protonation of E374 318 is likely required to allow HP2 closure, which completely occludes the

246 residues in a non-polar protein interior.

\section{Author contribution and interest conflict}

249 B.Q. and O.B. designed the experiments; B.Q. performed the experiments, processed and analyzed 250 data, and refined the molecular models; D.M. optimized grid preparation and performed 251 preliminary data processing; D.M and B.Q. collected Cryo-EM data with Z.Y. overseeing all 252 aspects of the microscope operation; B.Q. and E.F. performed electrophysiology experiments; B.Q. 253 and O.B. wrote the manuscript with input from all authors.

\section{Competing interests}

256 The authors declare no competing financial interests. 
259 We thank the Weill Cornell Medicine mass spectrometry facility for verifying the protein identity

260 and the NYSBC Simons EM center for access to the microscopes. We thank Misha Kopylov at

261 NYSBC Simons EM center for assistance with data collection and Dr. Shengliu Wang from

262 MSKCC for the initial scripts for particle sorting. We thank Dr. Xiaoyu Wang and Didar Ciftci for

263 useful discussions.

\section{Data availability}

267 Atomic coordinates for the Cryo-EM structures have been deposited in the Protein Data Bank 268 under accession codes 6X2L, 6X2Z, 6X3E, and 6X3F. The corresponding Cryo-EM maps have 269 been deposited in the Electron Microscopy Data Bank (EMDB) under accession codes 22011, 270 22014, 22020, and 22021, respectively. Additional Cryo-EM maps have been deposited into 271 EMDB under accession codes 22022, 22023, and 22024. The other data that support the findings 272 of this study are available from the corresponding authors upon reasonable request. 


\section{Figures}

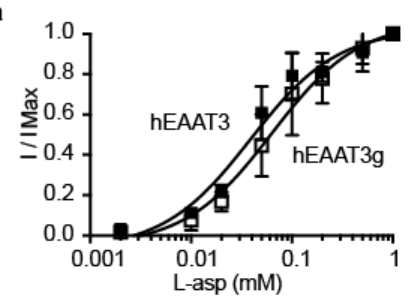

b

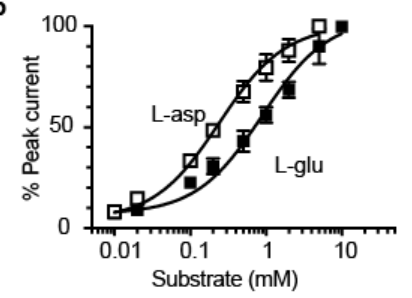

c

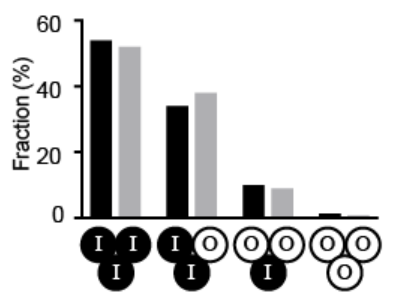

d
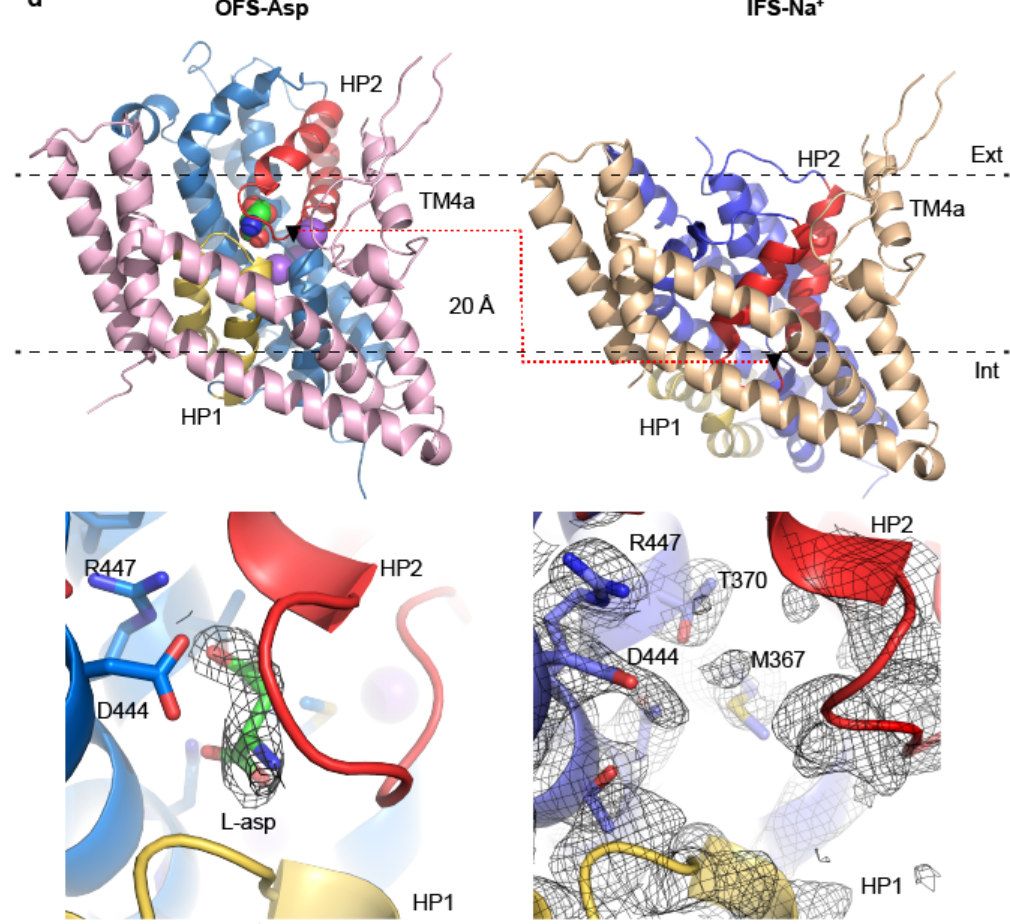

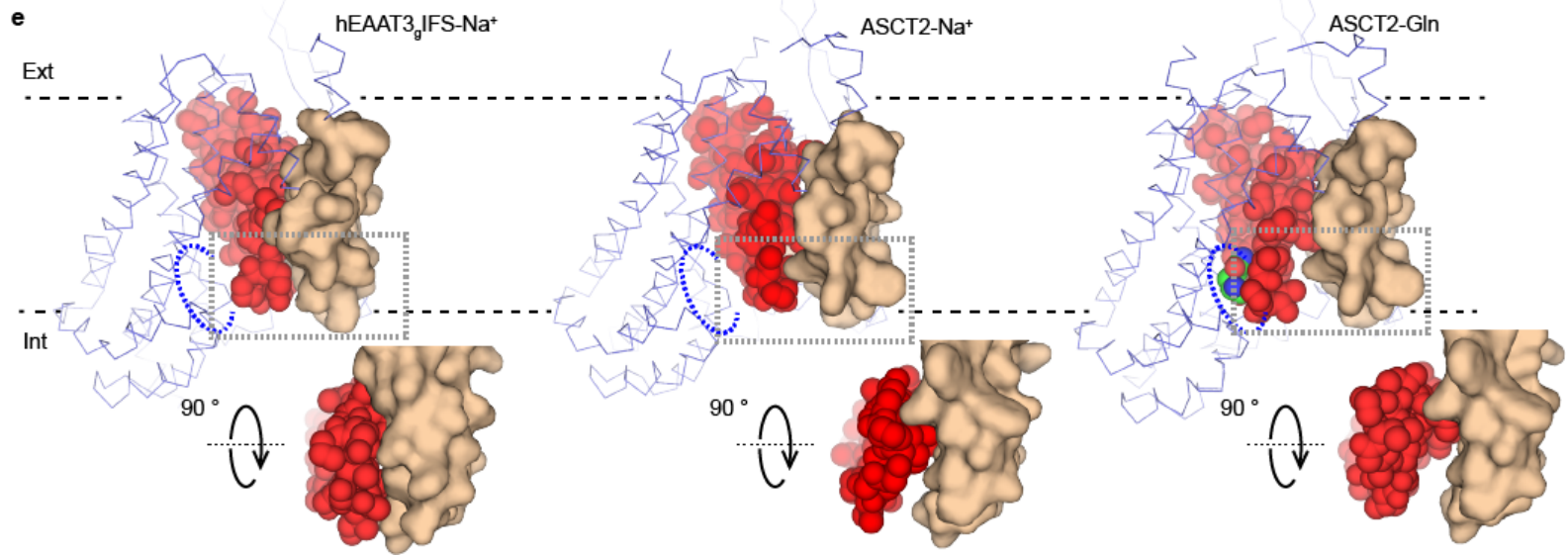

Figure 1. Alternating access mechanism of hEAAT3 $\mathrm{g}$. a, hEAAT3g (open squares) and wild type hEAAT3 (solid squares) show similar L-asp uptake in oocytes. b, hEAAT3g reconstituted into solid-supported membranes has a higher apparent affinity for L-asp (open squares) than L-glu (solid squares). c, Observed populations of hEAAT3g trimers with different configurations of protomers in the OFS (O) and IFS (I), as indicated below the graph (black columns), are consistent with binomial distributions with OFS probability of $19 \%$ (gray columns). The analysis is based on the Cryo-EM imaging data for hEAAT3g in the presence of $200 \mathrm{mM} \mathrm{NaCl}$ and $1 \mathrm{mM} \mathrm{L}$-asp. d, Structures of single hEAAT3g protomers in the IFS-Na ${ }^{+}$(left) and OFS-Asp (right) states. The scaffold domains are pink, and beige and the transport domains are in shades of blue. Structurally symmetric HP1 and HP2 are yellow and red, respectively. Substrate and ions are shown as spheres and colored by atom type. Below are the close-up views of the substrate-binding sites. The gray mesh objects are density maps contoured at $5.5 \sigma$ for L-asp (left) and $4.5 \sigma$ for the protein (right). e, Interactions between HP2 (red spheres) and scaffold domain (beige surface) for the inward- 
bioRxiv preprint doi: https://doi.org/10.1101/2020.06.01.127704; this version posted June 2, 2020. The copyright holder for this preprint (which was not certified by peer review) is the author/funder, who has granted bioRxiv a license to display the preprint in perpetuity. It is made available under aCC-BY-NC 4.0 International license.

facing hEAAT3g bound to $\mathrm{Na}^{+}$ions (left), ASCT2 bound to $\mathrm{Na}^{+}$ions (PDB accession code 6rvx, middle), and ASCT2-Gln bound to glutamine (PDB accession code 6gct, right). Dotted blue ellipses highlight the locations of the substrate-binding site for reference. The rest of the transport domains is shown as blue ribbons. The close-up views are below the panels. 

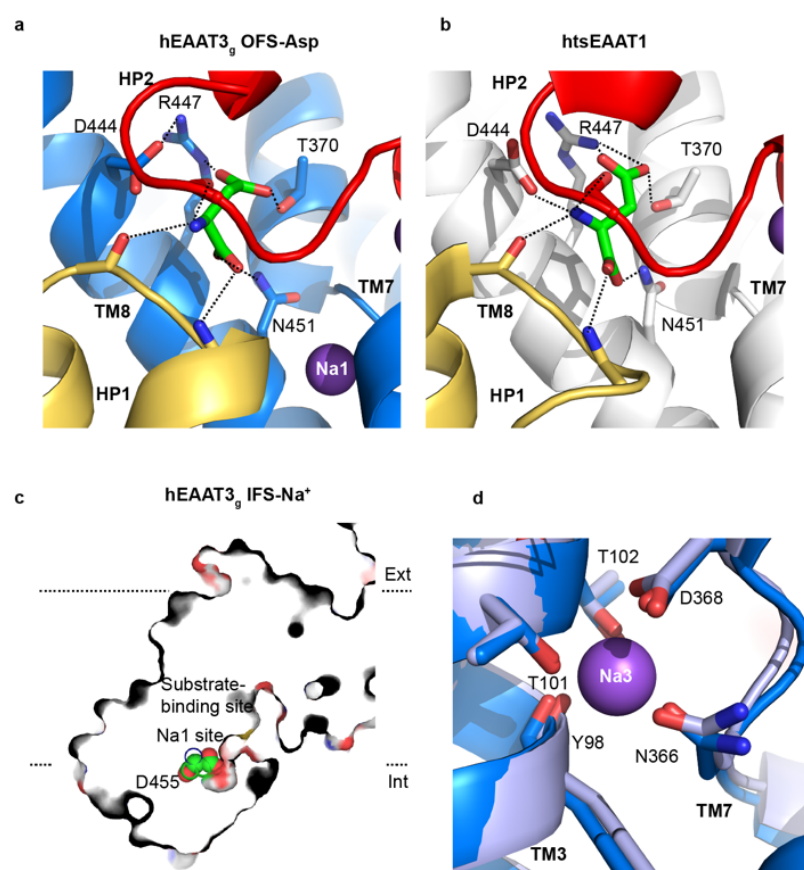

d
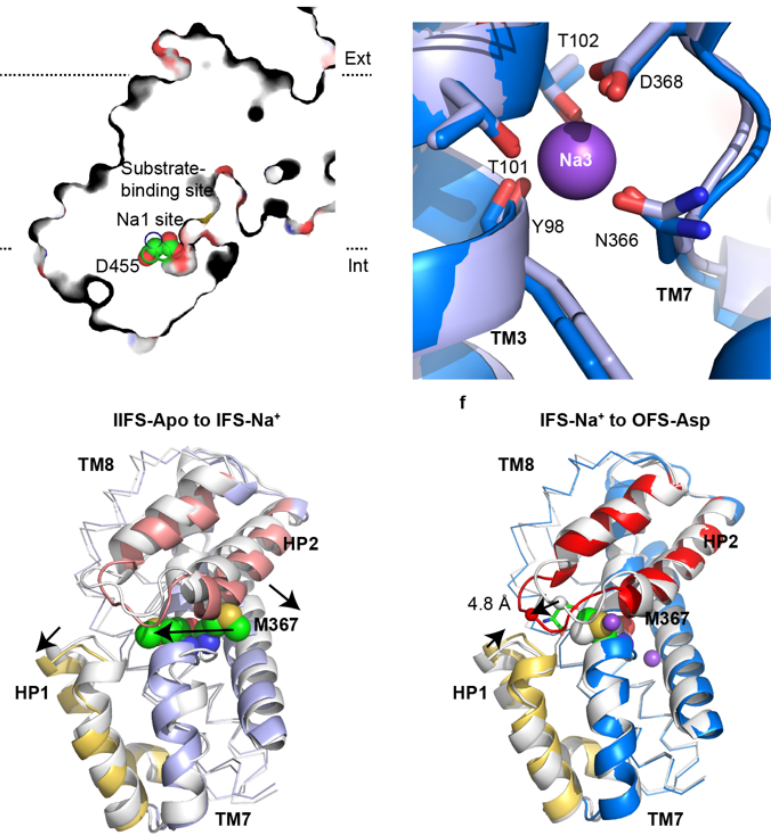

f

IFS-Na+ to OFS-Asp

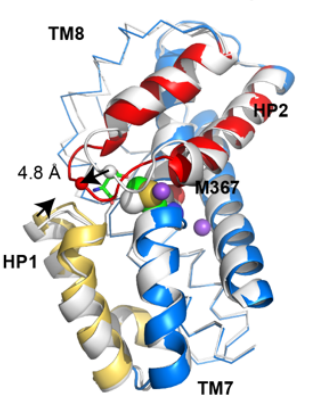

Figure 2. Substrate and sodium coupling in hEAAT3 $\mathrm{g}$. L-asp binds differently to hEAAT3g (a) and thermally stabilized human EAAT1 (htsEAAT1, PDB accession code 5lm4, b). Interactions between L-asp and EAATs are shown as dashed lines. HP1 and HP2 are colored yellow and red, respectively, L-asp green, TM 7 and TM 8 blue $\left(\mathrm{hEAAT} 3_{\mathrm{g}}\right.$ ) and white (htsEAAT1). c, Na1 is solvent-accessible. hEAAT3 $\mathrm{g}$ is shown as a thin slice taken through the Na1 site. The $\mathrm{Na}^{+}$-coordinating residue $\mathrm{D} 455$ is shown as spheres and colored by atom type. $\mathbf{d}$, a close-up view of the Na3 site in hEAAT3 ${ }_{g}$ OFS-Asp (marine) and IFS-Na ${ }^{+}$(light blue). e, f, Conformational changes within the transport domains transitioning from the IFS-Apo to IFS- $\mathrm{Na}^{+}$state (e) and from IFS- $\mathrm{Na}^{+}$to OFS-Asp state (f). The transport domains are white and shades of blue for the starting and ending states, respectively, with HP1 yellow, and HP2 salmon. M367 is shown as spheres and colored by atom type in all states. The arrows indicate conformational changes from IFS-Apo to IFS-Na ${ }^{+}$(e) and from IFS-Na ${ }^{+}$to OFS-Asp (f). 
a

OFS-Asp
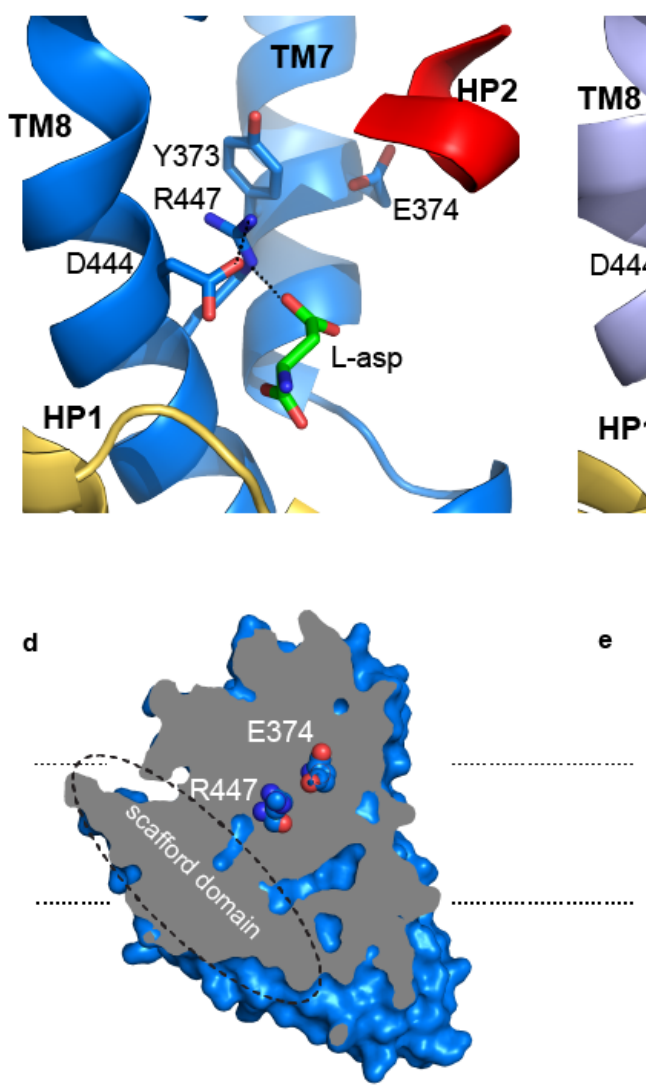

b

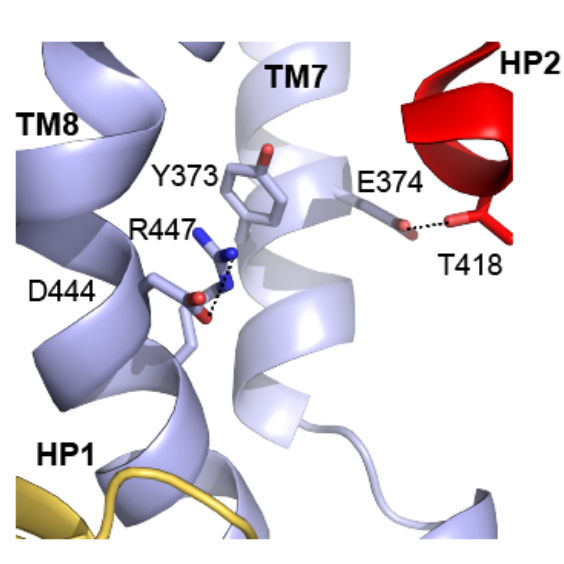

c

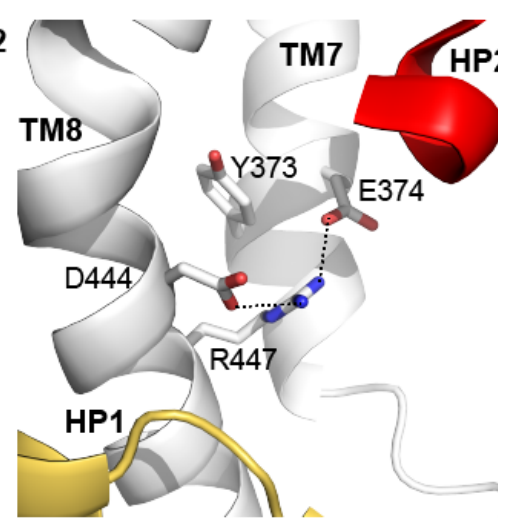

Figure 3. The proton coupling mechanism of hEAAT3. Close-up views of R447 and E374 in the OFS-Asp state (a), IFS-Na ${ }^{+}$state (b), and IFS-Apo state (c). Key interactions are shown as dashed lines. HP1 and HP2 are yellow and red, respectively. TMs 7 and 8 are marine, light blue, and white in a, b, and c, respectively. L-asp is green. The position of R447 and E374 relative to the membrane and their solvent accessibility in the OFS-Asp state (d), IFS$\mathrm{Na}^{+}(\mathbf{e})$, and IFS-Apo state (f). hEAAT3g is shown as a surface sliced approximately through the substrate-binding site and viewed in the membrane plane. Dashed ellipses mark the scaffold domains. R447 and E374 are shown as spheres. 


\section{Supplementary Methods}

\section{Protein expression and purification}

280 The codon-optimized full-length human EAAT3 was cloned into a modified pCDNA $3.1(+)$ 281 plasmid (Invitrogen), which has an N-terminal Strep tag-II followed by an enhanced green 282 fluorescent protein (eGFP) and PreScission protease site. Glycosylation sites, N178 and N195, 283 were mutated to threonine by site-directed mutagenesis. HEK293F cells (Invitrogen) at a density 284 of $2.5 \times 10^{6}$ cells / ml cultured in the FreeStyle ${ }^{\mathrm{TM}} 293$ medium (Gibco) were transiently transfected using $3 \mathrm{mg}$ of appropriate plasmid using poly-ethylenimine (PEI) (Polysciences) as a 1:3 plasmid to PEI weight ratio. The cells were diluted with an equal volume of fresh FreeStyle ${ }^{\mathrm{TM}} 293$ medium

2876 hours following the transfection, and valproic acid (Sigma) was added to a final concentration of $2882.2 \mathrm{mM}$ to boost protein expression $12 \mathrm{~h}$ later. The cells were collected $\sim 8$ hours after transfection 289 by centrifugation at $4,000 \mathrm{~g}$ for $10 \mathrm{~min}$ at $4^{\circ} \mathrm{C}$. Cell pellets were re-suspended in buffer containing 290 in mM 50 Tris-Cl pH 8.0, 1 L-asp, 1 EDTA, 1 TCEP, 1 PMSF, and 1:200 dilution of mammalian 291 protease inhibitor cocktail (Sigma). The re-suspended cells were disrupted using EmulsiFlex-C3 292 cell homogenizer (Avestin) or flash-frozen by liquid nitrogen and stored at $-80^{\circ} \mathrm{C}$ for further use. 293 Cell debris was removed by centrifugation at $10,000 \mathrm{~g}$ for $15 \mathrm{~min}$ at $4^{\circ} \mathrm{C}$, and membranes were 294 harvested by ultracentrifugation at $186,000 \mathrm{~g}$ for $1 \mathrm{~h}$ at $4^{\circ} \mathrm{C}$. The membrane pellets were resuspended and homogenized using a Dounce homogenizer in buffer containing in $\mathrm{mM} 50$ Tris-Cl $\mathrm{pH}$ 8.0, $200 \mathrm{NaCl}, 1$ aspartate, EDTA, 1 TCEP, 1 PMSF, 1:200 dilution of mammalian protease inhibitor cocktail, and 10\% (v:v) glycerol. The membranes were incubated with $2 \%$ dodecyl- $\beta$-Dmaltopyranoside (DDM, Anatrace) and 0.4\% cholesteryl hemisuccinate (CHS, Sigma) for $1 \mathrm{~h}$ at $4^{\circ} \mathrm{C}$. After ultracentrifugation at $186,000 \mathrm{~g}$ for $1 \mathrm{~h}$ at $4^{\circ} \mathrm{C}$ to remove insoluble material, the supernatant was incubated with Strep-Tactin sepharose resin (GE Healthcare) for $1 \mathrm{~h}$ at $4^{\circ} \mathrm{C}$. For

301 the L-asp containing samples, the resin was washed by 8 column volumes of buffer containing 50 $302 \mathrm{mM}$ Tris-Cl pH 8.0, $200 \mathrm{mM} \mathrm{NaCl}, 0.06 \%$ Digitonin.glyco-diosgenin (GDN, Anatrace), $1 \mathrm{mM}$ 303 TCEP, 5\% glycerol (wash buffer), and $1 \mathrm{mM}$ L-asp. Protein was eluted with 4 column volumes of 304 the wash buffer supplemented with $2.5 \mathrm{mM}$ D-desthiobiotin (elution buffer). Strep tag-II and eGFP 305 were removed by incubating the protein with homemade PreScission protease at 100:4 protein to 306 protease ratio at $4{ }^{\circ} \mathrm{C}$ overnight. EAAT3 was further purified by size exclusion chromatography using Superose 6 10/300 column (GE Healthcare) pre-equilibrated with $20 \mathrm{mM}$ Tris-Cl pH 8.0, 
$200 \mathrm{mM} \mathrm{NaCl}$, 1m M TCEP, 0.01\% GDN (gel filtration buffer) and either 1 or $20 \mathrm{mM}$ L-asp. To prepare hEAAT3 $\mathrm{g}$ in the presence of L-glu, L-asp in all buffers was replaced with $10 \mathrm{mM}$ L-glu. The peak fractions were pooled, and the protein was concentrated to $6 \mathrm{mg} / \mathrm{ml}$. To prepare $\mathrm{Na}^{+} / \mathrm{L}-$ asp-free samples, the protein was exchanged during size exclusion chromatography into a buffer containing $20 \mathrm{mM}$ Tris-Cl pH 8.0, $1 \mathrm{mM}$ TCEP, $0.01 \%$ GDN, and $250 \mathrm{mM} \mathrm{KCl}$ or $100 \mathrm{mM}$ choline

313 chloride.

\section{EM data acquisition}

316 To prepare grids for Cryo-EM imaging, 3-3.5 $\mu \mathrm{l}$ of EAAT3 at $\sim 6 \mathrm{mg} / \mathrm{ml}$ were applied to glow317 discharged QF R1.2/1.3 300 mesh gold or 400 mesh copper grids (Quantifoil, Großlöbichau,

318 Germany). Grids were either blotted for $3 \mathrm{~s}$ at $4^{\circ} \mathrm{C}$ and $100 \%$ set humidity and plunge-frozen into 319 liquid ethane using an FEI Mark IV Vitrobot (FEI company, part of Thermo Fisher Scientific, 320 Hillsboro, OR) or for 7 s using a Leica EM GP (Leica Microsystems Inc, Buffalo Grove, IL). Grids 321 were screened using an FEI Tecnai F20 TEM operated at $200 \mathrm{kV}$ through a Gatan 626 side-entry 322 cryo holder equipped with a Gatan K2 Summit direct detector (Gatan, Inc., Pleasanton, CA) or an 323 FEI Tecnai Spirit BioTWIN with a TVIPS F416 camera. The four here discussed Cryo-EM 324 datasets were collected at a $300 \mathrm{kV}$ FEI Titan Krios cryo-electron microscope (HHMI Janelia 325 Krios2) using SerialEM ${ }^{43,44}$. The microscope was equipped with a GIF Bioquantum energy filter 326 and a post-GIF K3 camera (Gatan, Inc.). A $100 \mu \mathrm{m} \mathrm{C} 2$ aperture, a $100 \mu \mathrm{m}$ objective aperture, and 327 a $20 \mathrm{eV}$ energy slit centered around the zero-loss peak were used during data collection. Dose 328 fractionation (movie) data were collected at a nominal magnification of $105,000 \mathrm{x}$, that is calibrated 329 magnification of $60096 \mathrm{x}$, corresponding to a physical pixel size of $0.832 \AA / \mathrm{px}(0.416 \AA / \mathrm{px}$ in the 330 resultant movie data with a binning of $0.5 \mathrm{x}$ selected). With the $\mathrm{K} 3$ camera in standard (non-CDS) 331 counted mode, a dose rate of $15 \mathrm{e} / \mathrm{px} / \mathrm{s}$ was employed, and each movie contains 60 frames with a 332 total accumulated dose of $60 \mathrm{e}^{-} / \AA^{2}$ on the sample. A nominal defocus range between $\sim-0.5$ to -1.5 $333 \mu \mathrm{m}$ was applied. The dataset in the presence of $20 \mathrm{mM}$ L-Asp was collected on a $300 \mathrm{kV}$ FEI Titan 334 Krios (NYSBC Krios2) equipped with a K2 Summit direct electron detector (Gatan, Inc.) at a 335 nominal magnification of $105,000 \mathrm{x}$, (calibrated magnification 45,620x) corresponding to a 336 physical pixel size of $1.096 \AA$ px. Each movie stack containing 50 frames was exposed in counting 
mode for $10 \mathrm{~s}\left(0.2 \mathrm{~s}\right.$ per frame) with a total dose of about $67.6 \mathrm{e}^{-} / \AA^{2}$. A nominal defocus range

338 between -1 to $-2 \mu \mathrm{m}$ was applied during data collection.

\section{Data processing}

340 For the dataset collected for EAAT3 in the presence of $1 \mathrm{mM} \mathrm{L-asp,} \mathrm{drift} \mathrm{correction} \mathrm{was} \mathrm{performed}$ 341 using MotionCor ${ }^{45}$, and the CTF parameters of micrographs were estimated using Gctf ${ }^{46}$. All 342 other steps of image processing were performed in Relion 3.0 $0^{47}$. 1,717,667 particles were selected 343 from 6,178 micrographs using a box of 288 pixels. The extracted particles were binned by two and 344 subjected to two rounds of $2 \mathrm{D}$ classification and one round of $3 \mathrm{D}$ classification. The $3 \mathrm{D}$ class 345 showing a good secondary structure was selected, and the particles were re-extracted using the 346 original pixel size of $0.832 \AA$.

After 3D refinement with C3 symmetry and post-processing, the resulting 3D reconstruction from 554,920 particles yielded a map at a $2.85 \AA$ global resolution. The particles after refinement were expanded using C3 symmetry, and an inverted mask generated using the inward-facing protomer was applied for signal subtraction (first signal subtraction). The resulting 1,664,760 protomers were subjected to 3D classification with a mask and without alignment. 325,222 particles in an outward-facing state and 1,339,538 particles in an inward-facing state were selected. The following 3D refinement and post-processing using the outward-facing particles yielded a map at $3.2 \AA$ resolution. An inverted mask generated using the outward-facing protomer was applied to the expanded particles for a second-round signal subtraction. 312,743 particles in an outward357 facing conformation were selected after masked 3D classification without alignment. A final map at $3.03 \AA$ resolution was obtained after 3D refinement and postprocessing.

We identified trimers with three outward-facing protomers (3o) and trimers with one outwardfacing protomer and two inward-facing protomers (2i1o) by analyzing the coordinates of the protomers generated by the 1st round of signal subtraction and 3D classification using a homewritten script. In total, 7,377 and 190,599 trimers were extracted from the raw micrographs, respectively. A final map at $3.69 \AA$ resolution was obtained for the 30 particles after refinement 365 and postprocessing. The first round of refinement of the 2 ilo particles yielded a map at $3.99 \AA$ resolution. The refined particles were then subjected to an additional 3D classification. 158,349 
particles were selected and further refined, yielding the final $2 \mathrm{i} 1 \mathrm{lo}$ map at $3.42 \AA$ resolution after postprocessing.

Other data sets were processed in Relion 3.0. Drift correlation was performed using Motioncor2 within Relion 3.0, and CTF parameters of micrographs were estimated using CTFFIND ${ }^{48}$. From the dataset collected for EAAT3 in $\mathrm{KCl}$ buffer, 1,769,994 particles were auto-picked and extracted with two-fold binning from 7,036 micrographs. 668,093 particles were selected and re-extracted after two rounds of $2 \mathrm{D}$ classification and one round of $3 \mathrm{D}$ classification. A final map at $2.85 \AA$ resolution was obtained after $3 \mathrm{D}$ refinement, postprocessing, and polishing. For the dataset collected in $10 \mathrm{mM}$ L-glutamate, 2,884,157 particles were auto-picked from 9,780 micrographs, and 647,481 particles were selected and re-extracted as above. After 3D refinement, postprocessing, and polishing, a map at $2.96 \AA \AA$ resolution was obtained. From the dataset collected in choline chloride buffer, 1,918,723 particles were auto-picked from 4,473 micrographs, and 435,398 particles were selected and re-extracted. A map at $3.24 \AA$ resolution was obtained after 3D refinement and post-processing. Further CTF-refinement and post-processing improved the alignment was also performed on all datasets as described above. No conformational heterogeneity was found. For the dataset collected in the presence of $20 \mathrm{mM}$ L-Asp, 1,533,624 particles were auto-picked from 3,424 micrographs using a box of 220 pixels. The particles were extracted using the original pixel size of $1.096 \AA$ and subjected to two rounds of $2 \mathrm{D}$ classification, and one round of 3D classification. One class showing a good secondary structure (168,777 particles) was selected. Following 3D reconstitution, polishing, and CTF refinement, a map at $3.71 \AA$ resolution was obtained.

\section{Model building and refinement}

Model building was carried out in COOT $^{49}$, and the protein models were refined using Phenix ${ }^{50}$.

392 For cross-validation, the final model was displaced and then refined against the first unfiltered

393 half-map. FSC curves were calculated between the refined model and the first unfiltered half-map,

394 the refined model and the second unfiltered half-map (which was not refined), and the refined 395 model and the summed map. The well overlaid FSC curves of the two unfiltered maps indicated no over-fitting. The structure figures were prepared in Chimera ${ }^{51}$ or Pymol (DeLano Scientific). 


\section{Proteoliposome reconstitution}

398 Liposomes were prepared using 5:5:2 (w:w) ratio of 1-palmitoyl-2-oleoyl-sn-glycero-3399 phosphocholine (POPC), 1-palmitoyl-2-oleoyl-sn-glycero-3-phosphoethanolamine (POPE, Avanti

400 Polar Lipids) and CHS. The lipids in chloroform were dried and rehydrated at $20 \mathrm{mg} / \mathrm{ml}$ by 10 401 freeze-thaw cycles in buffer containing $50 \mathrm{mM}$ HEPES-Tris buffer, $\mathrm{pH}$ 7.4. The liposomes were 402 diluted to $4 \mathrm{mg} / \mathrm{ml}$ in buffer containing $50 \mathrm{mM}$ HEPES/ $\mathrm{NaOH}, \mathrm{pH} 7.4,200 \mathrm{mM} \mathrm{NaCl}, 1 \mathrm{mM}$ 403 TCEP and 1mM L-asp and extruded 11 times through $400 \mathrm{~nm}$ polycarbonate membranes (Avanti 404 Polar Lipids) using a syringe extruder (Avanti Polar Lipids) to form unilamellar liposomes. 405 Liposomes were destabilized with DDM at a 1:1.5 (w:w) lipid to the detergent ratio for $15 \mathrm{~min}$ at 406 room temperature, and the purified EAAT3 protein was added to the mixture at a 1:10 (w:w) 407 protein to lipid ratio for $30 \mathrm{~min}$ at room temperature. The detergent removal was carried out by 408 adding $100 \mathrm{mg} / \mathrm{ml}$ Bio-Beads SM-2 (Bio-Rad) for $30 \mathrm{~min}$ at room temperature followed by 5 409 additional rounds at $4^{\circ} \mathrm{C}$. To exchange the internal buffer, the proteoliposomes were pelleted by 410 ultracentrifugation at $100,000 \mathrm{~g}$ for $45 \mathrm{~min}$ at $4^{\circ} \mathrm{C}$, diluted into the desired buffer, and subjected to 411 two freeze-thaw cycles. Centrifugation and freeze-thaw steps were repeated three times. The 412 proteoliposomes were extruded 11 times through $400 \mathrm{~nm}$ polycarbonate membranes for immediate 413 use.

\section{Solid supported membrane (SSM) assay}

415 The internal proteoliposome resting buffer contained $50 \mathrm{mM} \mathrm{HEPES} / \mathrm{KOH}, \mathrm{pH}$ 7.4, $150 \mathrm{mM} \mathrm{KCl,}$ $4162 \mathrm{mM} \mathrm{MgCl}_{2}$. The sensors for SSM assay were prepared according to the instrument manual, and 417 the transport-coupled current was recorded by a double-solution exchange method ${ }^{52,53}$. Briefly, a 418 non-activating buffer containing $50 \mathrm{mM}$ HEPES/NaOH, pH 7.4, $150 \mathrm{mM} \mathrm{NaCl}, 2 \mathrm{mM} \mathrm{MgCl} 2$ was 419 flown through the sensor to build the ionic gradients across the membranes of the proteoliposomes 420 deposited on the sensor. The transport-coupled current was initiated by flowing non-activating 421 buffer supplemented with different concentrations of the substrate (activating buffers). Finally, the 422 sensor was rinsed in a resting buffer to restore the sensor. The L-asp and L-glu affinities hEAAT3 423 were measured using sensors from three independent proteoliposomes preparations, and the data 424 were fitted by the equation: 


$$
I=I_{\max } \frac{X}{K_{m}+X}+B
$$

Where $I$ is the current measure in the presence of different substrate concentrations, $I_{\max }$ is the fitted maximum current, $\mathrm{X}$ is the substrate concentration, and $B$ is the baseline offset. TFB-TBOA inhibition was carried out by adding the inhibitor to final concentrations of 10,3 , or $1 \mu \mathrm{M}$ to nonactivating buffer and activating buffer containing $100 \mu \mathrm{M}$ L-asp. Following recording the transport-activating current, the sensor was restored as described above.

\section{cRNA preparation, voltage-clamp oocyte recordings, and analysis}

434 Wild type hEAAT3 and hEAAT3 $\mathrm{g}_{\mathrm{g}}$ were cloned in a pTLN vector and transcribed in vitro using 435 the mMessage mMachine SP6 Kit (Thermo Fisher Scientific). Xenopus laevis oocytes were 436 purchased from Ecocyte Bio Science (Austin, TX, USA), injected with 25 ng of cRNA, and kept 437 at $18^{\circ} \mathrm{C}$ in $50 \%$ Leibovitz medium, $250 \mathrm{mg} / 1$ gentamycin, $1 \mathrm{mM}$ L-glutamine, $10 \mathrm{mM}$ HEPES pH

438 7.6. Glass microelectrodes were pulled with the resistance of 0.5-3 M $\Omega$ and backfilled with $3 \mathrm{M}$ $439 \mathrm{KCl}$. Currents were recorded $48 \mathrm{~h}$ for the wild type and $72 \mathrm{~h}$ for $\mathrm{hEAAT} 3_{\mathrm{g}}$ after injection in ND96 440 solution (96 mM NaCl, $2 \mathrm{mM} \mathrm{KCl}, 1.8 \mathrm{mM} \mathrm{CaCl}_{2}, 1 \mathrm{mM} \mathrm{MgCl}_{2}, 5 \mathrm{mM}$ HEPES pH 7.5 using an 441 OC-725C voltage-clamp amplifier (Warner Instruments, Hamden, CT). The data were acquired 442 with the Patchmaster (HEKA Elektronik, Lambrecht, Germany) at 5kHz, filtered with Frequency 443 Devices 8-pole Bessel filter at a corner frequency of $2 \mathrm{kHz}$ and analyzed using Ana (M. Pusch, 444 Istituto di Biofisica, Genova) and Prism (GraphPad). The stimulation protocol for IV curves was 445 as follows: from a holding potential of $-30 \mathrm{mV}$, the voltage was stepped to a variable voltage in 20 $446 \mathrm{mV}$ incremental jumps from $-100 \mathrm{mV}$ to $+60 \mathrm{mV}$ for $200 \mathrm{~ms}$ and stepped back to $-30 \mathrm{mV}$. For 447 calculating $K_{m}$, a $-100 \mathrm{mV}$ pulse was applied for $200 \mathrm{~ms}$ at different ligand concentrations. Each 448 concentration point was proceeded by $0 \mathrm{mM} \mathrm{L-asp}$ to control for changes in oocyte leak currents 449 and followed by $1 \mathrm{mM}$ to control for rundown or vice versa. Only oocytes that displayed delta 450 currents bigger than $600 \mathrm{nA}$ in the presence of saturating concentration of ligand were used. Each 451 point in dose-response curves was calculated as follows: (current - leak) / (current at $1 \mathrm{mM}$ L-asp $452-$ leak). 
bioRxiv preprint doi: https://doi.org/10.1101/2020.06.01.127704; this version posted June 2, 2020. The copyright holder for this preprint (which was not certified by peer review) is the author/funder, who has granted bioRxiv a license to display the preprint in perpetuity. It is made available under aCC-BY-NC 4.0 International license. 
a
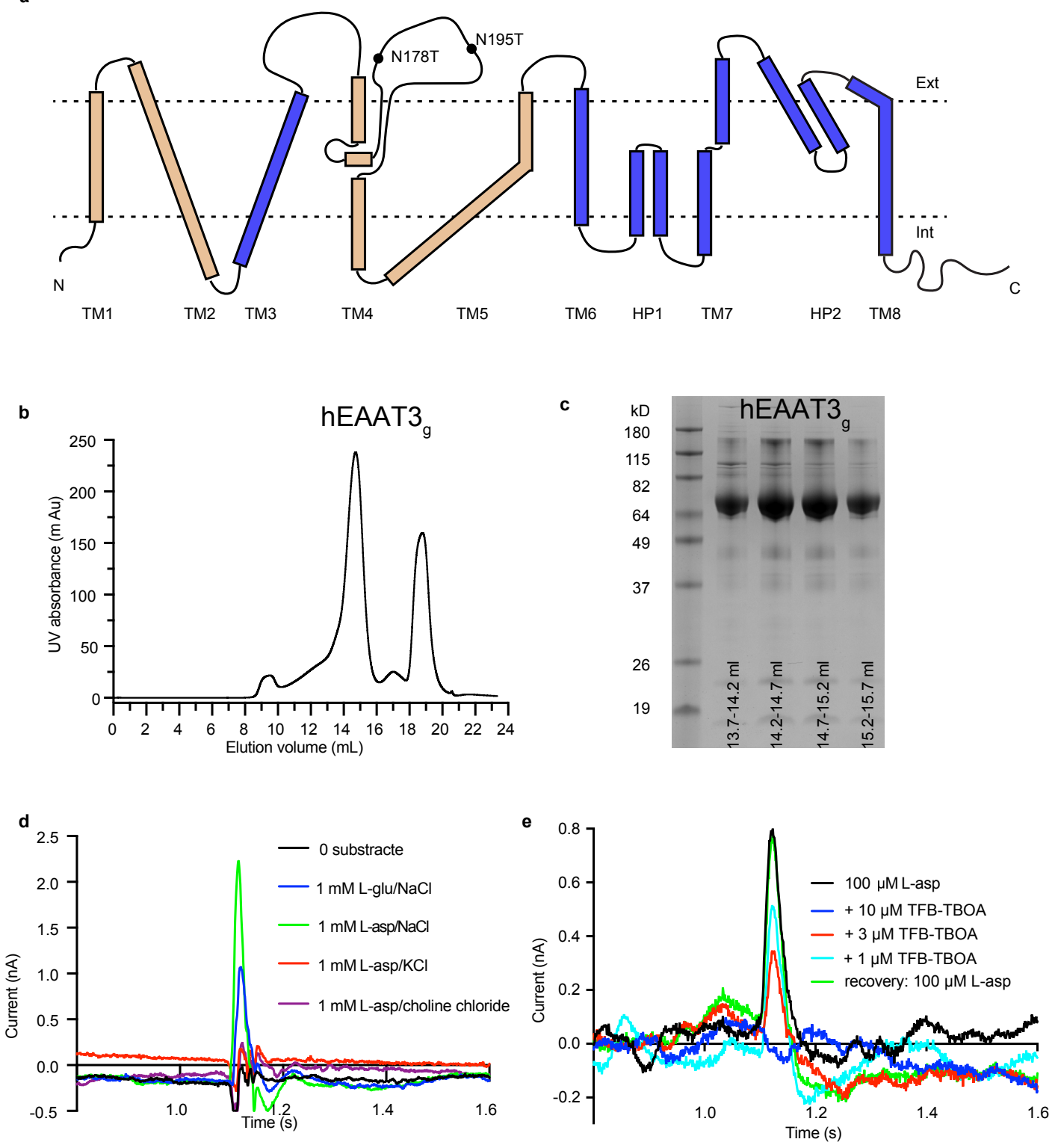

ED Figure 1. Purification and solid supported membrane assays of EAAT3g. a, Topology of hEAAT3g with the scaffold domain colored beige, and the transport domain blue. Black circles mark two glycosylation sites, N178 and N195, that were mutated to threonine. b, Size exclusion chromatography profile of hEAAT3g. c, SDS-PAGE analysis of hEAAT3g. d, Representative SSM recordings of hEAAT3 $\mathrm{g}$ in the presence of different ions (green, 1 $\mathrm{mM}$ L-asp and $150 \mathrm{mM} \mathrm{NaCl}$; blue, $1 \mathrm{mM}$ glutamate and $150 \mathrm{mM} \mathrm{NaCl}$; red, $1 \mathrm{mM}$-asp and $150 \mathrm{mM} \mathrm{KCl}$; purple, $150 \mathrm{mM}$ choline chloride; black, $150 \mathrm{mM} \mathrm{NaCl}$ ). The substrate transport currents are observed at $1.1 \mathrm{~s}$. Experiments were repeated on two independently prepared batches of proteoliposomes with similar results. e, Inhibition by TFB-TBOA of the transport currents induced by $100 \mu \mathrm{M}$ L-asp. Concentrations of TFB-TBOA were $10 \mu \mathrm{M}$ (blue), $3 \mu \mathrm{M}$ (red), and $1 \mu \mathrm{M}$ (cyan). The uninhibited current was measured before and after each addition of TFB-TBOA to monitor sensor activity. Only the first and last aspartate-induced currents are shown for clarity. 
bioRxiv preprint doi: https://doi.org/10.1101/2020.06.01.127704; this version posted June 2, 2020. The copyright holder for this preprint (which was not certified by peer review) is the author/funder, who has granted bioRxiv a license to display the preprint in perpetuity. It is made available under aCC-BY-NC 4.0 International license.

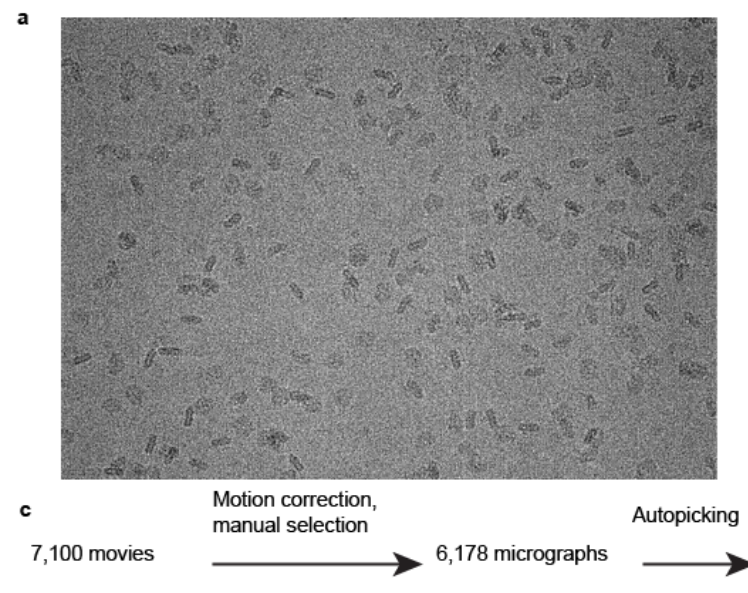

b
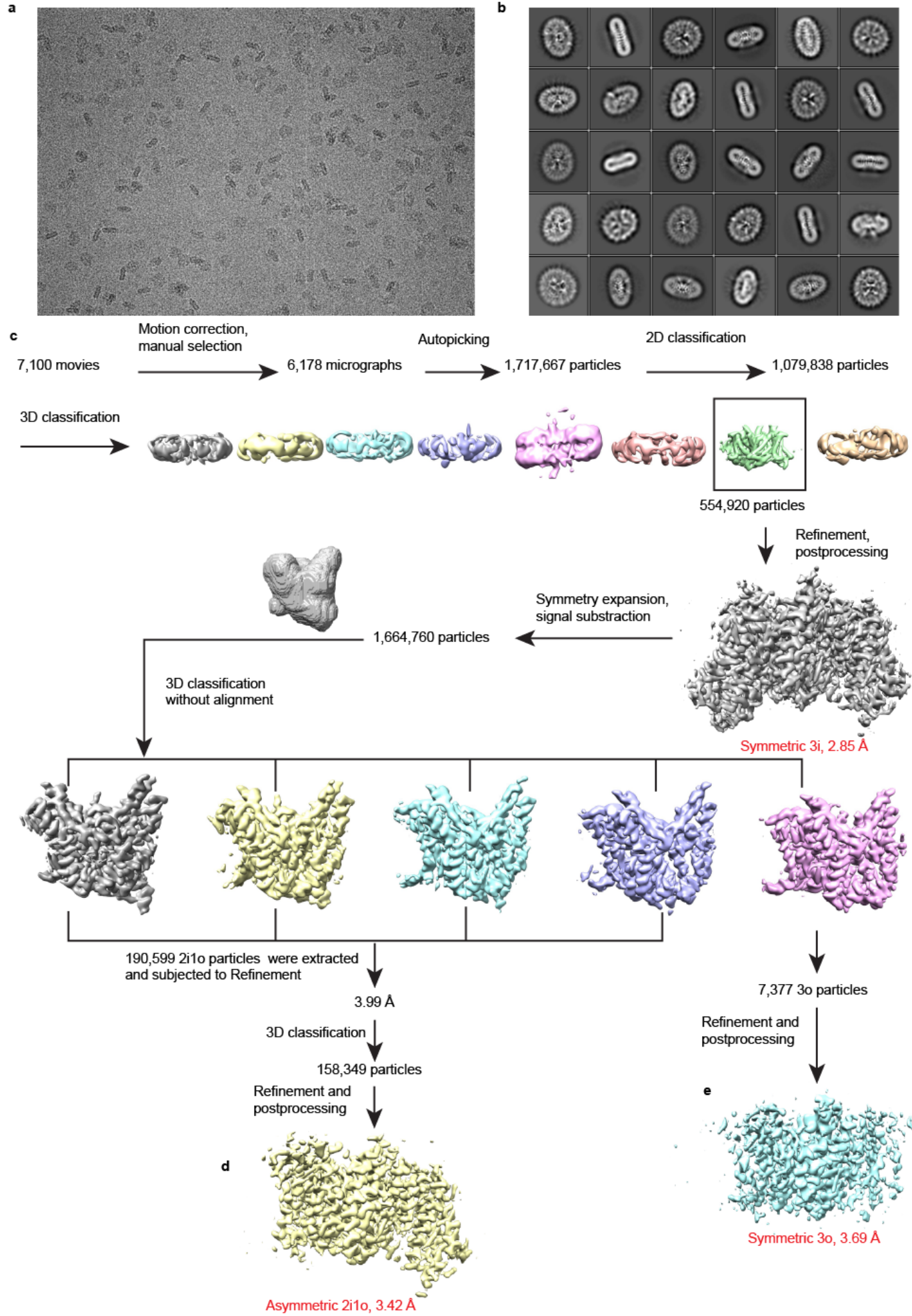

Symmetric 3i, $2.85 \AA$

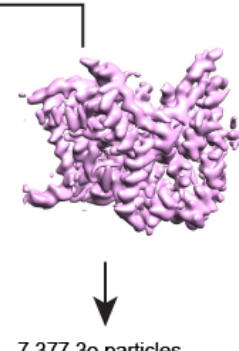

7,377 3o particles

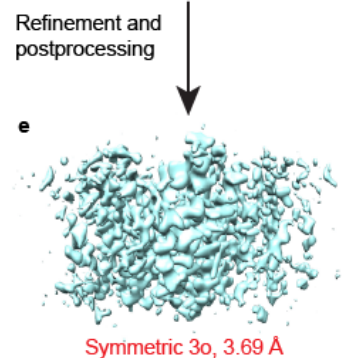

Asymmetric 2 i1o, $3.42 \AA$

ED Figure 2. Data processing for hEAAT3 $\mathrm{g}$ imaged in $200 \mathrm{mM} \mathrm{NaCl}$ and $1 \mathrm{mM} \mathrm{L-asp.} \mathrm{a,} \mathrm{Example} \mathrm{of} \mathrm{a}$ micrograph after motion correction. b, selected 2D class averages. c, Data processing flowchart. Following 3D classification, all particles were refined together using C3 symmetry to yield a map of an inward-facing symmetric 
bioRxiv preprint doi: https://doi.org/10.1101/2020.06.01.127704; this version posted June 2, 2020. The copyright holder for this preprint (which was not certified by peer review) is the author/funder, who has granted bioRxiv a license to display the preprint in perpetuity. It is made available under aCC-BY-NC 4.0 International license.

trimer, 3i. d, Symmetry expansion identified inward- and outward-facing protomers. Trimers containing two inward- and one outward-facing protomer were extracted and refined to yield a map for an asymmetric 2ilo trimer. e, Trimers containing all three outward-facing protomers were also extracted and refined using C 3 symmetry to yield a symmetric 30 map. 
bioRxiv preprint doi: https://doi.org/10.1101/2020.06.01.127704; this version posted June 2, 2020. The copyright holder for this preprint (which was not certified by peer review) is the author/funder, who has granted bioRxiv a license to display the preprint in perpetuity. It is made available under aCC-BY-NC 4.0 International license.

a

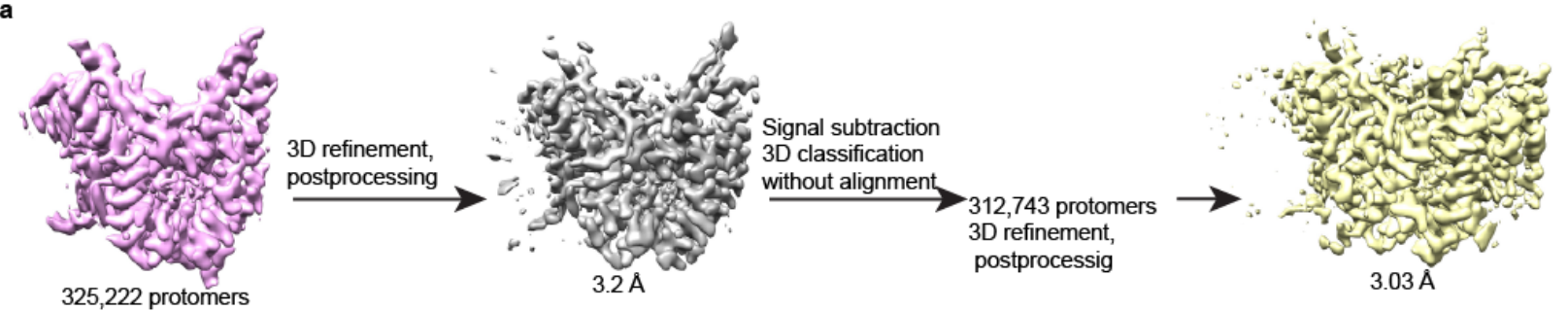

b

Symmetric 3i (IFS-Na+)
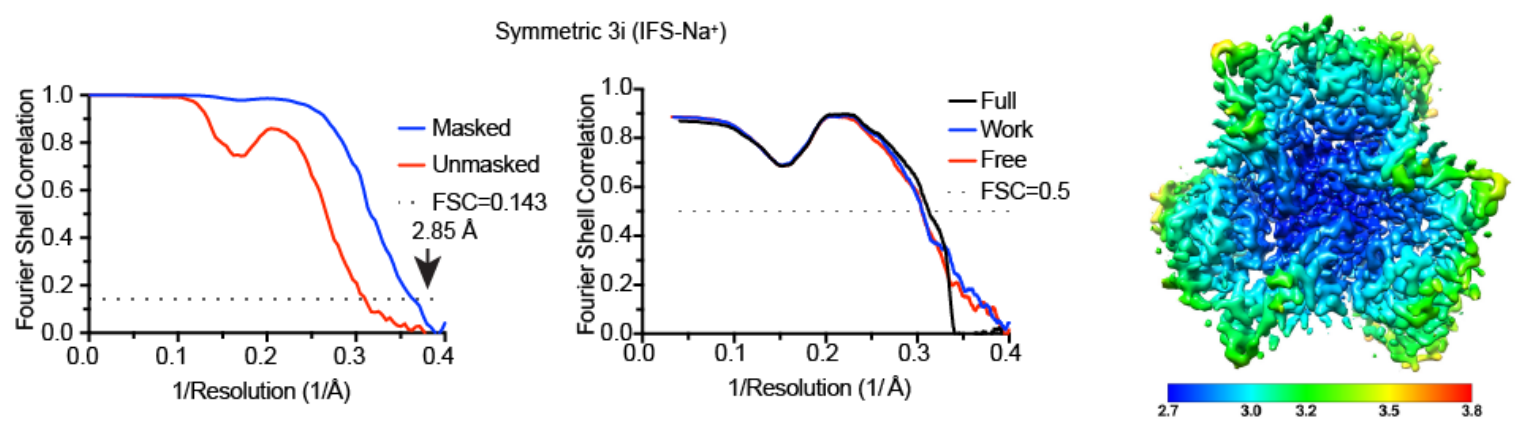

c
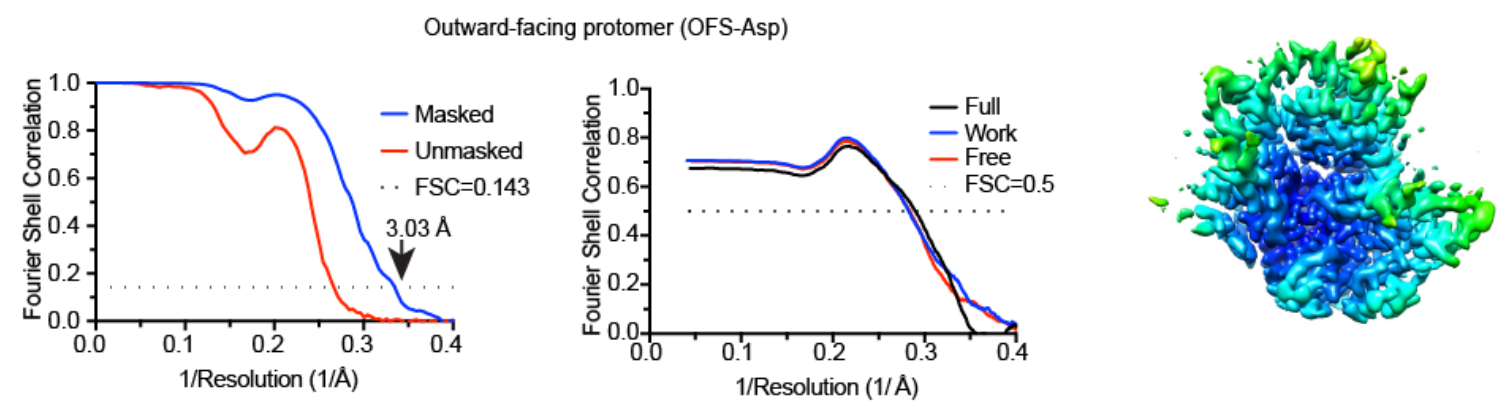

d

Asymmetric 2i10
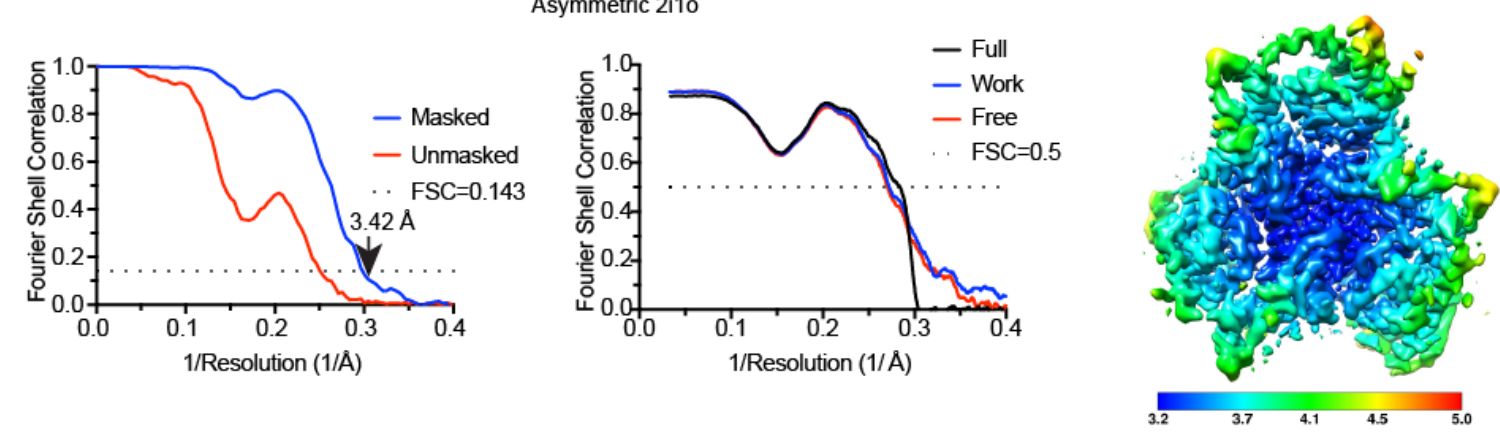

Symmetric inward-facing trimer in choline chloride (IFS-Apo)
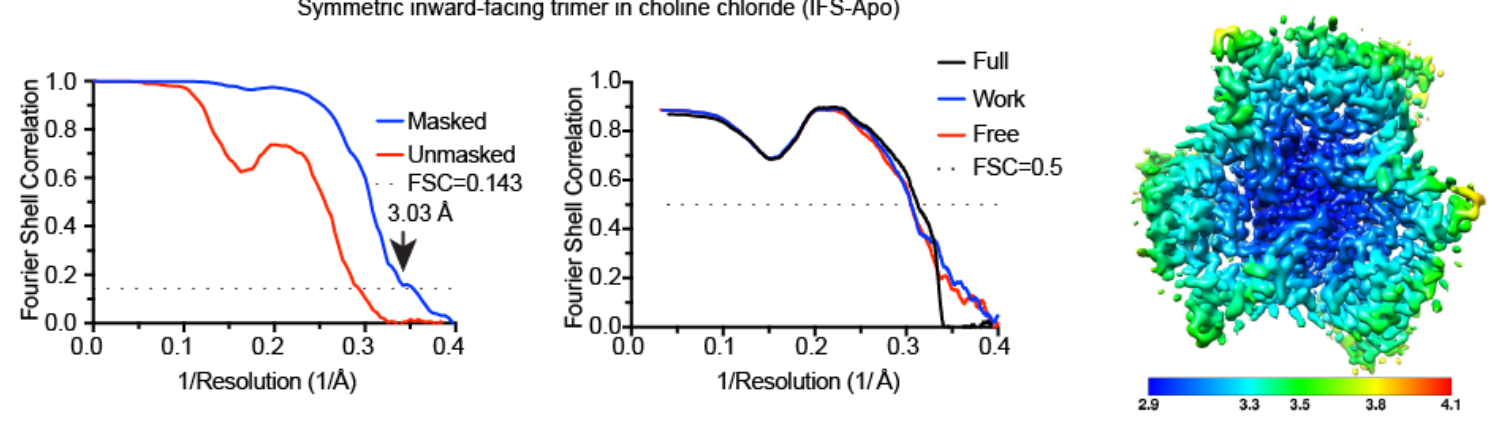
bioRxiv preprint doi: https://doi.org/10.1101/2020.06.01.127704; this version posted June 2, 2020. The copyright holder for this preprint (which

was not certified by peer review) is the author/funder, who has granted bioRxiv a license to display the preprint in perpetuity. It is made available under aCC-BY-NC 4.0 International license.

ED Figure 3. Flowchart of image processing (continued) and validation. a, 3D reconstructions of the outwardfacing protomer. b, c, d, and e, Fourier Shell Correlation (FSC) curves for the density maps (left); FSC curves of the refined model versus maps for cross-validation (middle); Density maps colored by the local resolution (right) of symmetric $3 \mathrm{i}$ IFS-Na ${ }^{+}(\mathbf{b})$, outward-facing protomer OFS-Asp (c), asymmetric 2ilo (d), and symmetric inwardfacing trimer imaged in choline chloride, IFS-Apo (e). 
bioRxiv preprint doi: https://doi.org/10.1101/2020.06.01.127704; this version posted June 2, 2020. The copyright holder for this preprint (which was not certified by peer review) is the author/funder, who has granted bioRxiv a license to display the preprint in perpetuity. It is made available under aCC-BY-NC 4.0 International license.

462
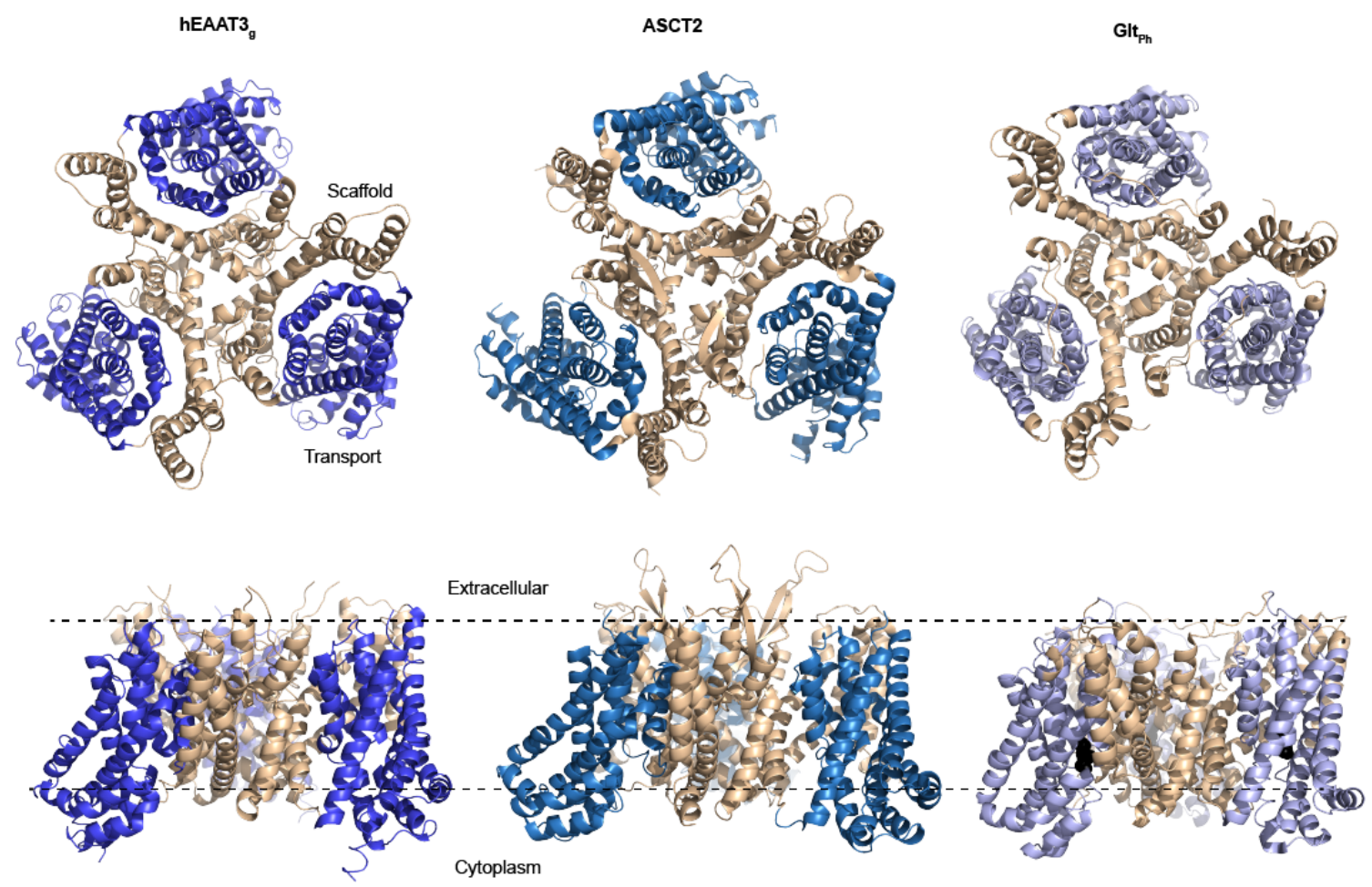

ED Figure 4. The overall architecture of hEAAT3 $\mathrm{g}$ resembles those of ASCT2 and Gitph. Inward-facing transporters are viewed from the extracellular space (top) or in the plane of the membrane (bottom). The transport and scaffold domains are colored beige and shades of blue, respectively. The PDB accession codes are 6rvx for 463 ASCT2 and 3kbc for Gltph. Black spheres correspond to the bound substrate L-asp in Gltph. 
a

IFS, $20 \mathrm{mM} \mathrm{L-asp}$

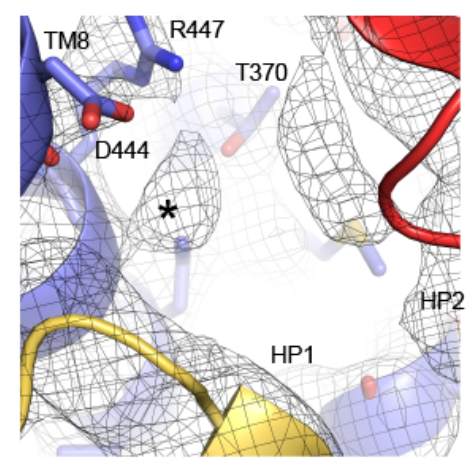

d

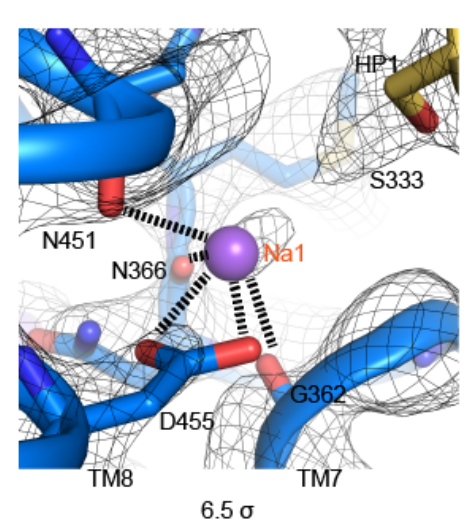

OFS-Asp b

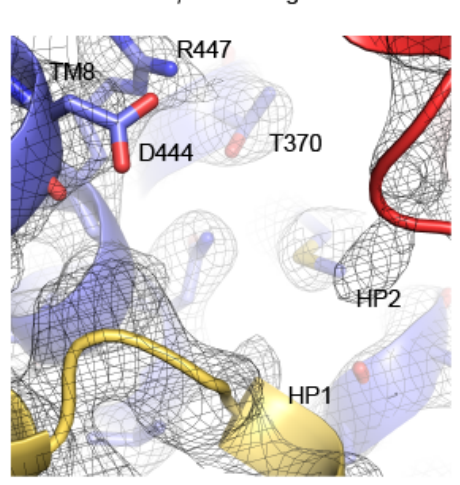

c

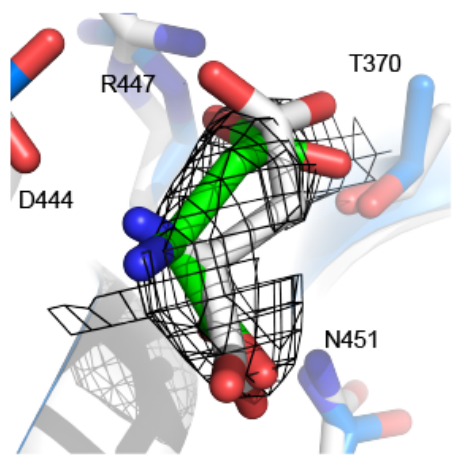

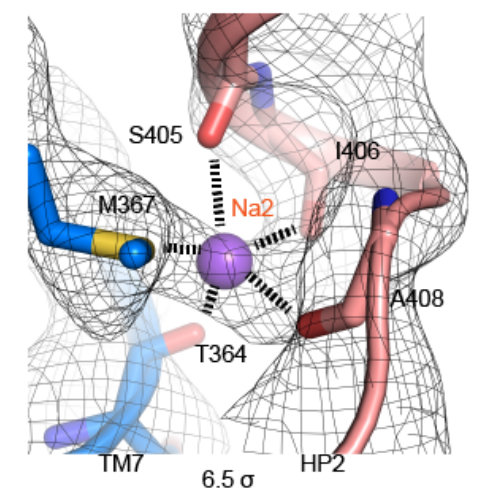

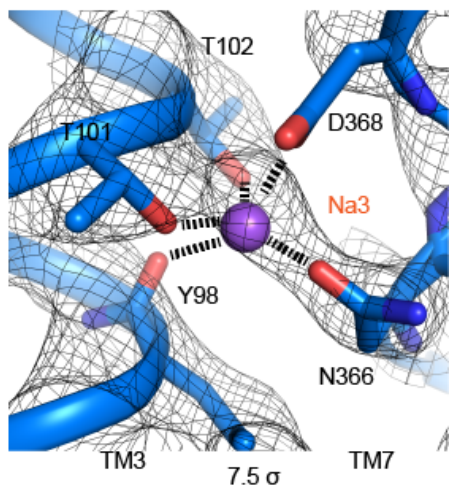

ED Figure 5. Density maps around the substrate and ion-binding sites. a and $\mathbf{b}$, Substrate-binding cavity of hEAAT $_{\mathrm{g}}$ in the inward-facing state in the presence of $20 \mathrm{mM} \mathrm{L}$-asp and $10 \mathrm{mM} \mathrm{L}$-glu, respectively. The black mesh shows the density maps. The black star in a marks an observed excess density, which might be due to partial occupancy of the substrate. The density around HP2 is fragmented, perhaps reflecting conformational heterogeneity. c, Superposition of the modeled L-asp in hEAAT3 $3_{\mathrm{g}}$ (green) and htsEAAT1 (white), showing that the rotamer observed in htsEAAT1 is not compatible with the excess density (black mesh) observed in hEEAT3g. d, $\mathrm{Na}^{+}$-binding sites in the OFS-Asp state of hEAAT3 $\mathrm{Na}^{+}$ions are shown as purple spheres, and their interactions with coordinating atoms are shown as dashed lines. The contour levels of the maps are below the panels. 

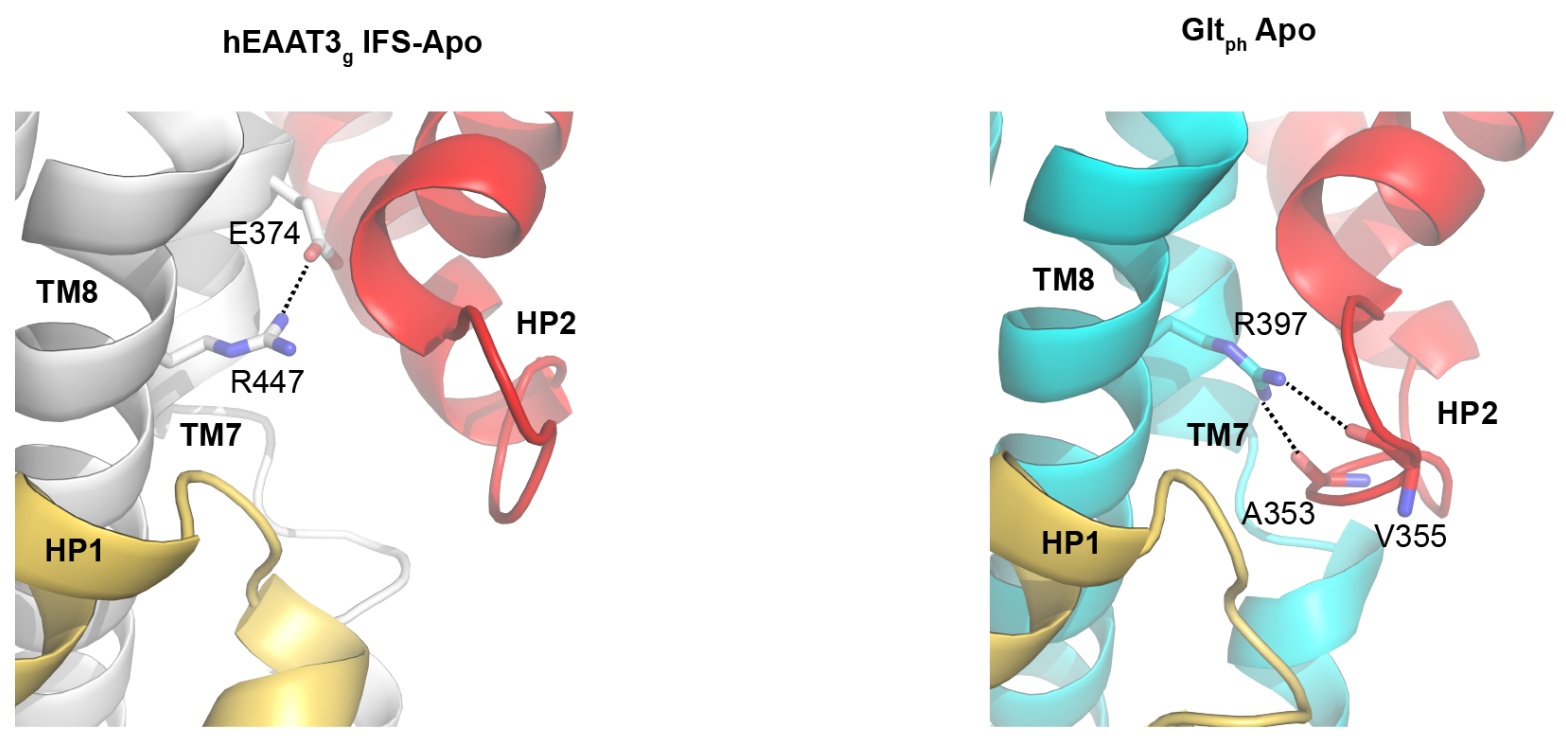

ED Figure 6. The substrate-binding site of the IFS-Apo hEAAT3 $g$ and inward-facing occluded apo Gitph (PDB accession code 4p19). Equivalent R447 in hEAAT3g and R397 in Gltph are shown as sticks along with their potential interaction partner residues. 
bioRxiv preprint doi: https://doi.org/10.1101/2020.06.01.127704; this version posted June 2, 2020. The copyright holder for this preprint (which was not certified by peer review) is the author/funder, who has granted bioRxiv a license to display the preprint in perpetuity. It is made available under aCC-BY-NC 4.0 International license.

469

a
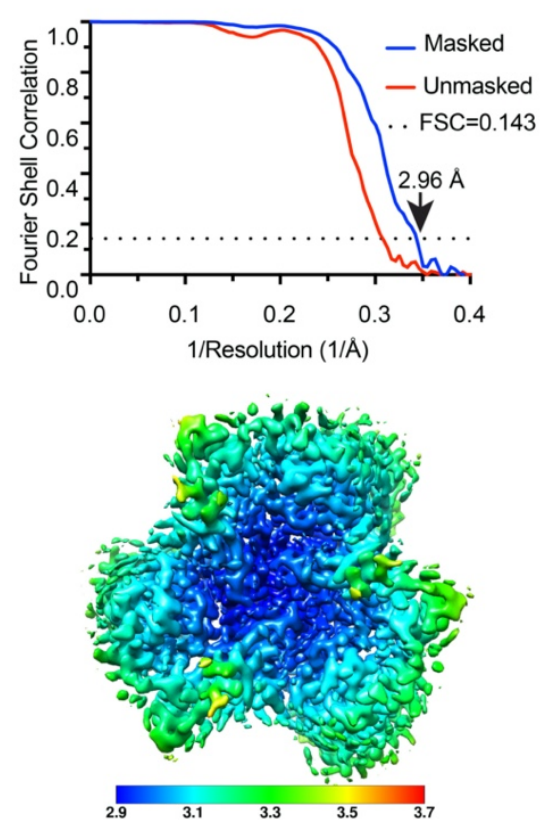

b
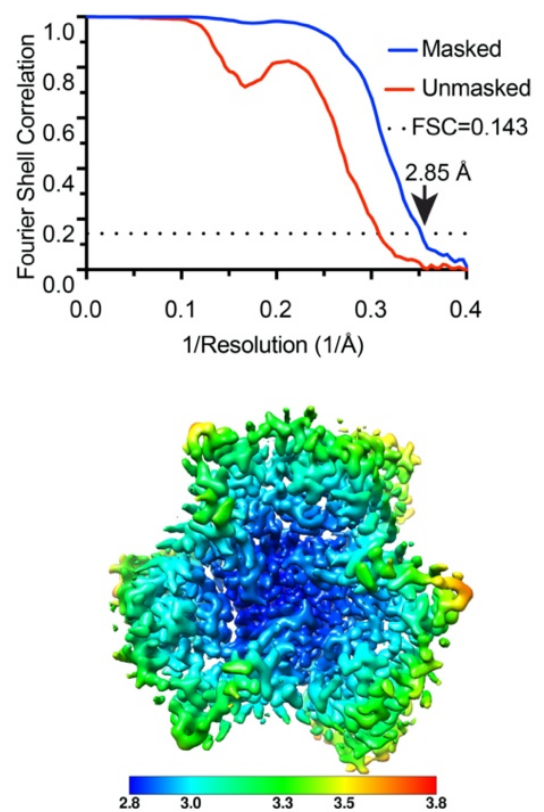

C

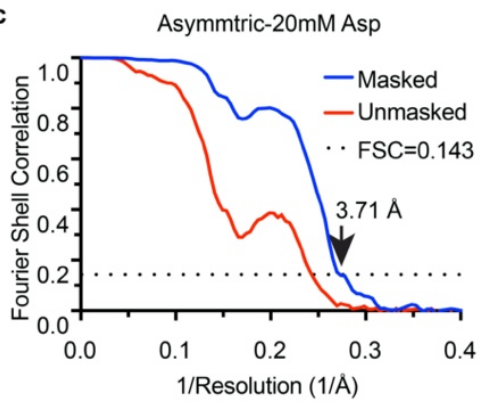

470

471

472

473

474
ED Figure 7. Cryo-EM data processing. Fourier Shell Correlation (FSC) curves for the density maps (top) and density maps colored by the local resolution (bottom) for a, IFS in $10 \mathrm{mM} \mathrm{L-Glu}$; $\mathbf{b}$, IFS in $250 \mathrm{mM} \mathrm{KCl}$; $\mathbf{c}$, asymmetric trimer, 2oli, in $20 \mathrm{mM} \mathrm{L-Asp.}$ 
Tabel 1: Cryo-EM data collection, refinement and validation statistics

\begin{tabular}{|c|c|c|c|c|}
\hline & $\begin{array}{l}{\mathrm{IFS}-\mathrm{Na}^{+}} \\
(\mathrm{EMDB}-22011) \\
(\mathrm{PDB}-6 \mathrm{X} 2 \mathrm{~L})\end{array}$ & $\begin{array}{l}\text { OFS-Asp } \\
(\text { EMDB-22014) } \\
(\text { PDB-6X2Z) }\end{array}$ & $\begin{array}{l}\text { Asymmetric, 2i1o } \\
\text { (EMDB-22020) } \\
\text { (PDB-6X3E) }\end{array}$ & $\begin{array}{l}\text { In-Apo } \\
(\text { EMDB-22021) } \\
(\text { PDB-6X3F) }\end{array}$ \\
\hline \multicolumn{5}{|l|}{$\begin{array}{l}\text { Data collection and } \\
\text { processing }\end{array}$} \\
\hline Magnification & 105,000 & 105,000 & 105,000 & 105,000 \\
\hline Voltage $(\mathrm{kV})$ & 300 & 300 & 300 & 300 \\
\hline Electron exposure $\left(\mathrm{e}-/ \AA^{2}\right)$ & 60 & 60 & 60 & 60 \\
\hline Defocus range $(\mu \mathrm{m})$ & -0.5 to -1.5 & -0.5 to -1.5 & -0.5 to -1.5 & -0.5 to -1.5 \\
\hline Pixel size $(\AA)$ & 0.832 & 0.832 & 0.832 & 0.832 \\
\hline Symmetry imposed & C3 & $\mathrm{C} 1$ & $\mathrm{C} 1$ & C3 \\
\hline Initial particle images (no.) & $1,717,667$ & $\begin{array}{l}1,664,760(\mathrm{C} 3 \\
\text { expand })\end{array}$ & 190,599 & $1,918,723$ \\
\hline Final particle images (no.) & 554,920 & $\begin{array}{l}312,743 \\
\text { protomer }\end{array}$ & 158,349 & 435,398 \\
\hline Map resolution $(\AA)$ & 2.85 & 3.03 & 3.42 & 3.03 \\
\hline FSC threshold & 0.143 & 0.143 & 0.143 & 0.143 \\
\hline Map resolution range $(\AA)$ & $23.96-2.66$ & $50-2.66$ & $34.24-3.15$ & $23.96-2.89$ \\
\hline \multicolumn{5}{|l|}{ Refinement } \\
\hline $\begin{array}{l}\text { Initial model used (PDB } \\
\text { code) }\end{array}$ & ab-initio & IFS-Na+ & $\begin{array}{l}\text { IFS-Na+ and } \\
\text { OFS-Asp }\end{array}$ & IFS-Na ${ }^{+}$ \\
\hline Model resolution $(\AA)$ & 2.88 & 3.36 & 3.45 & 3.15 \\
\hline FSC threshold & 0.5 & 0.5 & 0.5 & 0.5 \\
\hline Model resolution range $(\AA)$ & $29.17-2.88$ & $27.67-3.35$ & $27.28-3.45$ & $25.65-3.15$ \\
\hline $\begin{array}{l}\text { Map sharpening } B \text { factor } \\
\left(\AA^{2}\right)\end{array}$ & -136.18 & -122.27 & -137.77 & -121.29 \\
\hline \multicolumn{5}{|l|}{ Model composition } \\
\hline Non-hydrogen atoms & 9,396 & 3,205 & 9,466 & 9,366 \\
\hline Protein residues & 1,233 & 419 & 1,241 & 1,227 \\
\hline Ligands & & 4 & 4 & \\
\hline \multicolumn{5}{|l|}{$B$ factors $\left(\AA^{2}\right)$} \\
\hline Protein & 50.73 & 84.23 & 71.47 & 56.22 \\
\hline Ligand & & 95.04 & 87.02 & 64.47 \\
\hline \multicolumn{5}{|l|}{ R.m.s. deviations } \\
\hline Bond lengths $(\AA)$ & 0.009 & 0.005 & 0.006 & 0.006 \\
\hline Bond angles $\left(^{\circ}\right)$ & 0.974 & 0.830 & 0.899 & 0.925 \\
\hline \multicolumn{5}{|l|}{ Validation } \\
\hline MolProbity score & 1.16 & 1.60 & 1.27 & 1.51 \\
\hline Clashscore & 2.79 & 6.52 & 3.28 & 3.78 \\
\hline Poor rotamers (\%) & 0 & 0 & 0 & 0 \\
\hline \multicolumn{5}{|l|}{ Ramachandran plot } \\
\hline Favored (\%) & 97.53 & 96.37 & 97.14 & 95.04 \\
\hline Allowed (\%) & 2.47 & 3.63 & 2.86 & 4.96 \\
\hline Disallowed (\%) & 0 & 0 & 0 & 0 \\
\hline
\end{tabular}


bioRxiv preprint doi: https://doi.org/10.1101/2020.06.01.127704; this version posted June 2, 2020. The copyright holder for this preprint (which was not certified by peer review) is the author/funder, who has granted bioRxiv a license to display the preprint in perpetuity. It is made

Table 2:Cryo-EM data collection and processing

\begin{tabular}{llll}
\hline & $\begin{array}{l}\text { IFS in 10 mM L- } \\
\text { Glu } \\
(\text { EMDB-22022) }\end{array}$ & $\begin{array}{l}\text { IFS in 250 mM } \\
\text { KCl } \\
\text { (EMDB-22023) }\end{array}$ & $\begin{array}{l}\text { Asymmetric 2o1i } \\
\text { in 20 mM L-Asp } \\
\text { (EMDB-22024) }\end{array}$ \\
\hline $\begin{array}{l}\text { Data collection and } \\
\text { processing }\end{array}$ & & & \\
Magnification & 105,000 & 105,000 & 105,000 \\
Voltage $(\mathrm{kV})$ & 300 & 300 & 300 \\
Electron exposure $\left(\mathrm{e}-/ \AA^{2}\right)$ & 60 & 60 & 67.6 \\
Defocus range $(\mu \mathrm{m})$ & -0.5 to -1.5 & -0.5 to -1.5 & -1 to -2 \\
Pixel size $(\AA)$ & 0.832 & 0.832 & 1.096 \\
Symmetry imposed & $\mathrm{C} 3$ & $\mathrm{C} 3$ & $\mathrm{C} 1$ \\
Initial particle images $($ no.) & $2,884,157$ & $1,769,994$ & $1,533,624$ \\
Final particle images $($ no. $)$ & 647,481 & 668,093 & 168,777 \\
Map resolution $(\AA)$ & 2.96 & 2.85 & 3.71 \\
$\quad$ FSC threshold & 0.143 & 0.143 & 0.143 \\
Map resolution range $(\AA)$ & $18.43-2.89$ & $18.43-2.69$ & $24.11-3.40$ \\
\hline
\end{tabular}

481 


\section{References}

4831 Vandenberg, R. J. \& Ryan, R. M. Mechanisms of glutamate transport. Physiological reviews 93, 1621-1657, doi:10.1152/physrev.00007.2013 (2013).

2 Grewer, C., Gameiro, A. \& Rauen, T. SLC1 glutamate transporters. Pflugers Archiv : European journal of physiology 466, 3-24, doi:10.1007/s00424-013-1397-7 (2014).

3 Bjorn-Yoshimoto, W. E. \& Underhill, S. M. The importance of the excitatory amino acid transporter 3 (EAAT3). Neurochem Int 98, 4-18, doi:10.1016/j.neuint.2016.05.007 (2016).

4 Kanai, Y. \& Hediger, M. A. Primary structure and functional characterization of a highaffinity glutamate transporter. Nature 360, 467-471, doi:10.1038/360467a0 (1992).

5 Rothstein, J. D. et al. Localization of neuronal and glial glutamate transporters. Neuron 13, 713-725, doi:10.1016/0896-6273(94)90038-8 (1994).

6 Bailey, C. G. et al. Loss-of-function mutations in the glutamate transporter SLC1A1 cause human dicarboxylic aminoaciduria. J Clin Invest 121, 446-453, doi:10.1172/JCI44474 (2011).

7 Arnold, P. D., Sicard, T., Burroughs, E., Richter, M. A. \& Kennedy, J. L. Glutamate transporter gene SLC1A1 associated with obsessive-compulsive disorder. Arch Gen Psychiatry 63, 769-776, doi:10.1001/archpsyc.63.7.769 (2006).

8 Peghini, P., Janzen, J. \& Stoffel, W. Glutamate transporter EAAC-1-deficient mice develop dicarboxylic aminoaciduria and behavioral abnormalities but no neurodegeneration. The EMBO journal 16, 3822-3832, doi:10.1093/emboj/16.13.3822 (1997).

9 Holmseth, S. et al. The density of EAAC1 (EAAT3) glutamate transporters expressed by neurons in the mammalian CNS. $J$ Neurosci 32, 6000-6013, doi:10.1523/JNEUROSCI.5347-11.2012 (2012).

10 Rothstein, J. D. et al. Knockout of glutamate transporters reveals a major role for astroglial transport in excitotoxicity and clearance of glutamate. Neuron 16, 675-686, doi:10.1016/s0896-6273(00)80086-0 (1996).

11 Rakhade, S. N. \& Loeb, J. A. Focal reduction of neuronal glutamate transporters in human neocortical epilepsy. Epilepsia 49, 226-236, doi:10.1111/j.1528-1167.2007.01310.x (2008).

12 McCullumsmith, R. E. \& Meador-Woodruff, J. H. Striatal excitatory amino acid transporter transcript expression in schizophrenia, bipolar disorder, and major depressive disorder. Neuropsychopharmacology 26, 368-375, doi:10.1016/S0893-133X(01)00370-0 (2002).

13 Fujita, H., Sato, K., Wen, T. C., Peng, Y. \& Sakanaka, M. Differential expressions of glycine transporter 1 and three glutamate transporter mRNA in the hippocampus of gerbils with transient forebrain ischemia. Journal of cerebral blood flow and metabolism : official journal of the International Society of Cerebral Blood Flow and Metabolism 19, 604-615, doi:10.1097/00004647-199906000-00003 (1999).

14 Bianchi, M. G., Bardelli, D., Chiu, M. \& Bussolati, O. Changes in the expression of the glutamate transporter EAAT3/EAAC1 in health and disease. Cell Mol Life Sci 71, 20012015, doi:10.1007/s00018-013-1484-0 (2014).

15 Zerangue, N. \& Kavanaugh, M. P. Interaction of L-cysteine with a human excitatory amino acid transporter. The Journal of physiology 493 ( Pt 2), 419-423, doi:10.1113/jphysiol.1996.sp021393 (1996). 
52616 Watts, S. D., Torres-Salazar, D., Divito, C. B. \& Amara, S. G. Cysteine transport through

527 excitatory amino acid transporter 3 (EAAT3). PloS one 9, e109245, doi:10.1371/journal.pone.0109245 (2014).

17 Aoyama, K. et al. Neuronal glutathione deficiency and age-dependent neurodegeneration in the EAAC1 deficient mouse. Nat Neurosci 9, 119-126, doi:10.1038/nn1609 (2006).

18 Zerangue, N. \& Kavanaugh, M. P. Flux coupling in a neuronal glutamate transporter. Nature 383, 634-637, doi:10.1038/383634a0 (1996).

19 Watzke, N., Bamberg, E. \& Grewer, C. Early intermediates in the transport cycle of the neuronal excitatory amino acid carrier EAAC1. J Gen Physiol 117, 547-562, doi:10.1085/jgp.117.6.547 (2001).

20 Borre, L. \& Kanner, B. I. Coupled, but not uncoupled, fluxes in a neuronal glutamate transporter can be activated by lithium ions. The Journal of biological chemistry 276, 40396-40401, doi:10.1074/jbc.M104926200 (2001). by the neuronal excitatory amino acid carrier 1. J Gen Physiol 116, 609-622, doi:10.1085/jgp.116.5.609 (2000).

22 Zhang, Z. et al. Transport direction determines the kinetics of substrate transport by the glutamate transporter EAAC1. Proceedings of the National Academy of Sciences of the United States of America 104, 18025-18030, doi:10.1073/pnas.0704570104 (2007).

23 Yernool, D., Boudker, O., Jin, Y. \& Gouaux, E. Structure of a glutamate transporter homologue from Pyrococcus horikoshii. Nature 431, 811-818, doi:10.1038/nature03018 (2004).

24 Boudker, O., Ryan, R. M., Yernool, D., Shimamoto, K. \& Gouaux, E. Coupling substrate and ion binding to extracellular gate of a sodium-dependent aspartate transporter. Nature 445, 387-393, doi:10.1038/nature05455 (2007).

25 Reyes, N., Ginter, C. \& Boudker, O. Transport mechanism of a bacterial homologue of glutamate transporters. Nature 462, 880-885, doi:10.1038/nature08616 (2009).

26 Guskov, A., Jensen, S., Faustino, I., Marrink, S. J. \& Slotboom, D. J. Coupled binding mechanism of three sodium ions and aspartate in the glutamate transporter homologue GltTk. Nat Commun 7, 13420, doi:10.1038/ncomms13420 (2016).

27 Canul-Tec, J. C. et al. Structure and allosteric inhibition of excitatory amino acid transporter 1. Nature 544, 446-451, doi:10.1038/nature22064 (2017).

28 Garaeva, A. A. et al. Cryo-EM structure of the human neutral amino acid transporter ASCT2. Nature structural \& molecular biology 25, 515-521, doi:10.1038/s41594-0180076-y (2018).

29 Yu, X. et al. Cryo-EM structures of the human glutamine transporter SLC1A5 (ASCT2) in the outward-facing conformation. eLife 8, doi:10.7554/eLife.48120 (2019).

30 Grewer, C. et al. Individual subunits of the glutamate transporter EAAC1 homotrimer function independently of each other. Biochemistry 44, 11913-11923, doi:10.1021/bi050987n (2005).

31 Leary, G. P., Stone, E. F., Holley, D. C. \& Kavanaugh, M. P. The glutamate and chloride permeation pathways are colocalized in individual neuronal glutamate transporter subunits. J Neurosci 27, 2938-2942, doi:10.1523/JNEUROSCI.4851-06.2007 (2007).

32 Koch, H. P., Brown, R. L. \& Larsson, H. P. The glutamate-activated anion conductance in excitatory amino acid transporters is gated independently by the individual subunits. $J$ Neurosci 27, 2943-2947, doi:10.1523/JNEUROSCI.0118-07.2007 (2007). 
33 Garaeva, A. A., Guskov, A., Slotboom, D. J. \& Paulino, C. A one-gate elevator mechanism for the human neutral amino acid transporter ASCT2. Nat Commun 10, 3427, doi:10.1038/s41467-019-11363-x (2019).

34 Arkhipova, V., Guskov, A. \& Slotboom, D. J. Structural ensemble of a glutamate transporter homologue in lipid nanodisc environment. Nat Commun 11, 998, doi:10.1038/s41467-020-14834-8 (2020).

35 Bendahan, A., Armon, A., Madani, N., Kavanaugh, M. P. \& Kanner, B. I. Arginine 447 plays a pivotal role in substrate interactions in a neuronal glutamate transporter. The Journal of biological chemistry 275, 37436-37442, doi:10.1074/jbc.M006536200 (2000).

36 Teichman, S., Qu, S. \& Kanner, B. I. Conserved asparagine residue located in binding pocket controls cation selectivity and substrate interactions in neuronal glutamate transporter. The Journal of biological chemistry 287, 17198-17205, doi:10.1074/jbc.M112.355040 (2012).

37 Verdon, G., Oh, S., Serio, R. N. \& Boudker, O. Coupled ion binding and structural transitions along the transport cycle of glutamate transporters. eLife 3, e02283, doi:10.7554/eLife.02283 (2014).

38 Wang, X. \& Boudker, O. Large domain movements through lipid bilayer mediate substrate release and inhibition of glutamate transporters. bioRxiv, 2020.2005.2019.103432,

39 Grewer, C., Watzke, N., Rauen, T. \& Bicho, A. Is the glutamate residue Glu-373 the proton

40 Olsson, M. H., Sondergaard, C. R., Rostkowski, M. \& Jensen, J. H. PROPKA3: Consistent

41 Akyuz, N., Altman, R. B., Blanchard, S. C. \& Boudker, O. Transport dynamics in a glutamate transporter homologue. Nature 502, 114-118, doi:10.1038/nature12265 (2013). Grewer, C., Watzke, N., Wiessner, M. \& Rauen, T. Glutamate translocation of the neuronal

43 Mastronarde, D. N. Automated electron microscope tomography using robust prediction

44 Schorb, M., Haberbosch, I., Hagen, W. J. H., Schwab, Y. \& Mastronarde, D. N. Software glutamate transporter EAAC1 occurs within milliseconds. Proceedings of the National Academy of Sciences of the United States of America 97, 9706-9711, doi:10.1073/pnas.160170397 (2000).

615

616

45 Zheng, S. Q. et al. MotionCor2: anisotropic correction of beam-induced motion for improved cryo-electron microscopy. Nature methods 14, 331-332, doi:10.1038/nmeth.4193 (2017).

46 Zhang, K. Getf: Real-time CTF determination and correction. Journal of structural biology 193, 1-12, doi:10.1016/j.jsb.2015.11.003 (2016).

47 Scheres, S. H. RELION: implementation of a Bayesian approach to cryo-EM structure determination. Journal of structural biology 180, 519-530, doi:10.1016/j.jsb.2012.09.006 (2012). 
61748 Rohou, A. \& Grigorieff, N. CTFFIND4: Fast and accurate defocus estimation from 618 electron micrographs. Journal of structural biology 192, 216-221, 619 doi:10.1016/j.jsb.2015.08.008 (2015).

62049 Emsley, P., Lohkamp, B., Scott, W. G. \& Cowtan, K. Features and development of Coot. Acta crystallographica. Section D, Biological crystallography 66, 486-501, doi:10.1107/S0907444910007493 (2010).

50 Adams, P. D. et al. PHENIX: a comprehensive Python-based system for macromolecular structure solution. Acta crystallographica. Section D, Biological crystallography 66, 213221, doi:10.1107/S0907444909052925 (2010).

51 Pettersen, E. F. et al. UCSF Chimera--a visualization system for exploratory research and analysis. J Comput Chem 25, 1605-1612, doi:10.1002/jcc.20084 (2004).

52 Bazzone, A., Barthmes, M. \& Fendler, K. SSM-Based Electrophysiology for Transporter Research. Methods Enzymol 594, 31-83, doi:10.1016/bs.mie.2017.05.008 (2017).

53 Krause, R., Watzke, N., Kelety, B., Dorner, W. \& Fendler, K. An automatic Methods 177, 131-141, doi:10.1016/j.jneumeth.2008.10.005 (2009). 